\title{
Call admission control with heterogeneous mobile stations in cellular/WLAN interworking systems
}

\author{
Hyung-Taig Lim, Younghyun Kim, Sangheon Pack and Chul-Hee Kang
}

\begin{abstract}
Although different call admission control (CAC) schemes have been proposed for cellular-wireless local area network (WLAN) interworking systems, no studies consider mobile stations (MSs) only with a single interface (for either WLANs or cellular networks) and thus these MSs will experience higher call blocking and dropping probabilities. In this article, we propose a new CAC scheme that considers both the MSs with a single interface and with dual interfaces. By employing the concept of guard-bands, the proposed CAC scheme gives higher priority to MSs with a single interface than those with dual interfaces to accommodate more MSs. The call blocking and dropping probabilities are analyzed using Markov chains and how to determine appropriate guard bands for CAC is investigated through cost minimization problems. Analytical and simulation results demonstrate that the proposed scheme can achieve lower blocking probabilities compared with existing schemes that do not include single interface MSs.
\end{abstract}

Keywords: call admission control, WLAN, cellular, heterogeneous mobile stations, performance analysis

\section{Introduction}

Recently, different types of wireless networks, such as cellular networks, worldwide interoperability for microwave access (WiMAX), and wireless local area networks (WLANs), have widely been deployed. These wireless networks have quite different characteristics; for instance, cellular networks provide ubiquitous coverage with low bandwidth whereas WLANs provide high data rates at cheap cost but can only provide lower mobility. In fact, none of these wireless networks can satisfy the wide ranging requirements from diverse users and this is the key motivation for integrating these heterogeneous wireless networks for providing users with the best connectivity $(\mathrm{ABC})$ at all times [1].

Extensive work has been done in the integration of heterogeneous networks [2-9] and to allow seamless mobility across these heterogeneous networks (i.e., vertical handoff), two integration architectures, having both tightly coupled and loosely coupled architectures, have been introduced in $[2,3]$. In [4,5], vertical handover decisions where an mobile station (MS) selects the most

\footnotetext{
* Correspondence: shpack@korea.ac.kr

School of Electrical Engineering, Korea University, Seoul, Korea
}

appropriate network to avoid unnecessary handovers and wastages of resource have been proposed. The resource allocation in heterogeneous wireless networks has been investigated in [6-9]; the study in [6] investigates the admission control strategies for the data traffic in a hierarchical system consisting of macrocell and microcell layers; the authors of [7] introduce the first WLAN scheme and analyze its performance; Song et al. [8] determine an admission control scheme in which MSs try to access networks with specific probabilities for the maximum number of users; and Stevens-Navarro et al. [9] introduce an admission control scheme for multi-services. In these previous studies, it is assumed that all MSs have dual interfaces to cellular/WLAN systems and they can access both systems even though it is obvious that some MSs have only one interface, either cellular or WLAN. Therefore, the existing call admission control (CAC) schemes may lead to an "unfair" situation because they treat single- and dual-interface MSs equally. Specifically, WLAN-only and cellular-only MSs can be admitted to only WLAN and cellular networks, respectively, and therefore they experience higher call blocking/dropping probabilities than MSs with dual 
interfaces. Consequently, it is necessary to give higher priority to WLAN-only or cellular-only MSs for CAC in cellular/WLAN systems.

In this article, a new CAC scheme is proposed that considers heterogeneous types of MSs. In the proposed scheme, a well-known guard channel scheme is examined to give high priority to both the handover MSs and the single interfaced MSs. For performance evaluation, analytical models based on Markov chains are developed to analyze the call blocking and the call dropping probabilities. Furthermore, the optimal allocation of the guard channels is investigated by formulating cost minimization problems. Analytical and simulation results are presented which demonstrate that the new proposed scheme can achieve lower call blocking and call dropping probabilities than existing schemes because cellular- or WLAN-only MSs have higher priorities than the dual-interfaced MSs.

The rest of the article is organized as follows. Sections 2 and 3 describe the system model and the proposed CAC scheme considering heterogeneous MSs. Section 4 analyzes the performance of the CAC scheme through Markov chains. Section 5 presents numerical results and Section 6 describes the main conclusions from the research presented in the article.

\section{System model}

As shown in Figure 1, we consider a network architecture where a WLAN hotspot is overlaid within a cell. It is assumed that the WLAN hotspot is not located at the boundary of the cell. In the WLAN coverage, MSs with both WLAN and cellular interfaces can access both systems and therefore the WLAN coverage area is referred to as a "double coverage" area. On the other hand, the region outside the WLAN is denoted as a "cellular only"

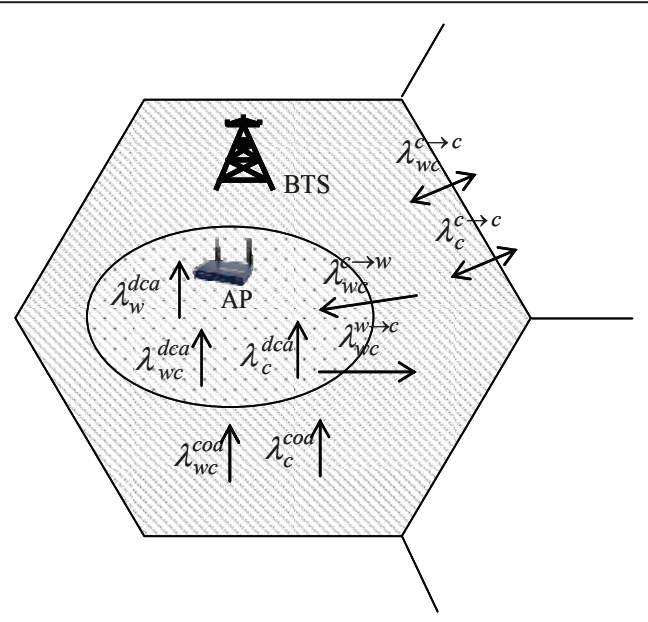

Figure 1 An integrated cellular/WLAN system model area [7-9], i.e., a cell area consists of a double coverage area and a cellular only area. Hereinafter, 'dca', 'coa', and 'ca' stand for double coverage areas, cellular only areas, and cell areas, respectively. Table 1 lists the important notations used in this article.

We consider three types of MSs, namely, WLAN-only, cellular-only, and dual-interfaced MSs. A WLAN-only MS has only a WLAN radio interface and the calls from the WLAN-only MS (i.e., WLAN-only calls) cannot be serviced in the cellular only area. On the other hand, a cellular-only MS has only a cellular radio interface and the calls originated from the cellular-only MS (i.e., cellular-only calls) can be accepted only by the cellular network even in double coverage areas. A dual-interfaced MS with WLAN and cellular interfaces can clearly access both the WLAN and the cellular network interfaces in the double coverage areas. Hence, we refer to the calls from dual-interfaced MSs as dual access calls. Also, we assume that dual-interfaced MSs can be accepted only in either the WLAN or the cellular network at a time, that is, we do not consider that the dual-interfaced MSs simultaneously use both networks for traffics. To consider these heterogeneous types of MSs, call requests need to be classified into WLAN-only calls, cellular-only calls, and dual access calls. In the proposed scheme, it is assumed that the types of MSs are provisioned to a certain server such as home location register (HLR) and the CAC entity can obtain the types of MSs from the server or the types of MSs can be queried to MSs on receiving call requests. Throughout this article, 'c', 'w', and 'wc' stand for cellular-only, WLAN-only, and dual access calls, respectively.

Figure 1 illustrates the call arrival rates and the handoff rates in different areas. In this article, all call arrivals are assumed to follow Poisson distributions. We do not consider handoffs between two WLANs due to sparse deployments of WLANs, and therefore there exists only new calls from WLAN-only MSs whose arrival rates are denoted as $\lambda_{\mathrm{w}}^{\mathrm{dca}}$. New calls from cellular-only MSs can be generated in a double coverage area or a cellular only area, and their arrival rates are given by $\lambda_{c}^{\text {dca }}$ and $\lambda_{c}^{\text {coa }}$, respectively. On the other hand, the handoff rate of cellular-only MSs is denoted by $\lambda_{c}^{c \rightarrow c}$. A dual-interfaced MS can generate new calls in a double coverage area and in a cellular only area, and the arrival rates are given by $\lambda_{\mathrm{wc}}^{\mathrm{dca}}$ and $\lambda_{\mathrm{wc}}^{\mathrm{coa}}$, respectively. The horizontal handoff rate between two cells is $\lambda_{\mathrm{wc}}^{\mathrm{c} \rightarrow \mathrm{c}}$, whereas the vertical handoff rates from a WLAN to a cellular network (upward vertical handoff) and from a cellular network to a WLAN (downward vertical handoff) are denoted by $\lambda_{\mathrm{wc}}^{\mathrm{w} \rightarrow \mathrm{c}}$, and $\lambda_{\mathrm{wc}}^{\mathrm{c} \rightarrow \mathrm{w}}$, respectively.

We adopt the non-uniform mobility model within a single cell as in [7] where users in double coverage and 


\begin{tabular}{cc}
\hline Notation & Meaning \\
\hline$\lambda_{w}^{d c a}$ & Mean arrival rate of new WLAN-only calls in a double coverage area \\
$\lambda_{c}^{d c a}$ & Mean arrival rate of new cellular-only calls in a double coverage area \\
$\lambda_{c}^{c o a}$ & Mean arrival rate of new cellular-only calls in a cellular only area \\
$\lambda_{w c}^{d c a}$ & Mean arrival rate of new dual access calls in a double coverage area \\
$\lambda_{w c}^{c o a}$ & Mean arrival rate of new dual access calls in a cellular only area \\
$\lambda_{w c}^{c \rightarrow c}$ & Mean arrival rate of horizontal handoff dual access calls \\
$\lambda_{c}^{c \rightarrow c}$ & Mean arrival rate of horizontal handoff cellular only calls \\
$\lambda_{w c}^{w \rightarrow c}$ & Mean arrival rate of upward vertical handoff calls \\
$\lambda_{w c}^{c \rightarrow w}$ & Mean arrival rate of downward vertical handoff calls \\
$T^{d c a}$ & Residence time in a double coverage area \\
$T^{c o a}$ & Residence time in a cellular only area \\
$p^{c o a \rightarrow d c a}$ & Probability of a user moving from a cellular only area to a double coverage area \\
$p^{c o a \rightarrow c o a}$ & Cell residence time of dual access calls accepted by the cellular network in a double coverage area \\
$T_{v}^{c a}$ & Cell residence time of cellular only calls accepted by the cellular network in a double coverage area \\
$T_{w c, d c a}^{c a}$ & Cell residence time of cellular only calls accepted by the cellular network in a cellular only area \\
$T_{c, d c a}^{c a}$ &
\end{tabular}

cellular only areas have different mobility behaviors since WLAN hotspots are usually deployed in indoor environments and thus the users in the double coverage area have low mobility. Specifically, the residence time in the double coverage area, $T^{\mathrm{dca}}$, is assumed to follow an exponential distribution with mean $1 / \eta^{\text {dca }}$. On the other hand, the residence time in the cellular only area, $T^{\text {coa }}$, has an exponential distribution with mean $1 / \eta^{\text {coa }}$. As illustrated in Figure 2, the MSs moving out the WLAN coverage enter the cellular-only area. The MSs moving out of the cellular-only area can enter the double coverage area with probability $p^{\text {coa } \rightarrow \text { dca }}$, whereas they move to neighbor cells with the probability $p^{\mathrm{coa} \rightarrow \mathrm{coa}}[7]$. The MS entering the double coverage area from the cellular only area can move to cellular-only areas later.

The residence time of a WLAN-only MS within a WLAN area has the same distribution as $T^{\text {dca }}$. Dual access calls generated with the double coverage area can be admitted to either a WLAN or a cellular network.

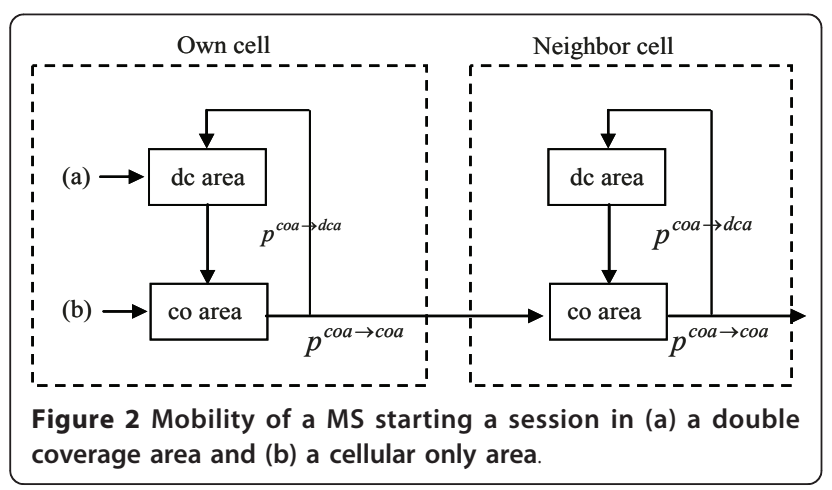

The residence time in the double coverage area of dual access calls admitted to the WLAN has the same distribution as $T^{\text {dca }}$. The dual access calls admitted to the cellular networks are assumed to stay in the cellular networks when vertical handoff requests to the WLAN are not allowed since dual-interfaced MSs can send call requests to the WLAN without breaking connections to the cellular networks. Hence, the residence time in the cell of dual access calls admitted to the cellular network in the double coverage area, $T_{\mathrm{wc}, \mathrm{dca}}^{\mathrm{ca}}$, can be expressed as $T_{w c, d c a}^{c a}=T_{1}^{c o a}+\cdots+T_{N_{w c, d c a}}^{d c a}+T_{N_{w c, c o a}}^{c o a}$, where $N_{w c, d c a}$ and $N_{w c}$, coa are the number of entrances of the double coverage area and the cellular only area until the MS moves out to neighbor cells or the calls are successfully accepted to WLAN through vertical handoff. Similarly, the cell residence time of the dual access calls originated in the cellular-only area, $T_{w c, c o a}^{c a}$, can be expressed as $T_{\mathrm{wc}, \mathrm{coa}}^{\mathrm{ca}}=T_{1}^{\mathrm{coa}}+\cdots+T_{N_{\mathrm{wc}, \mathrm{dca}}^{\mathrm{dca}}}^{\mathrm{ca}}+T_{N_{\mathrm{wc}, \mathrm{coa}}^{\mathrm{coa}}}^{\mathrm{coa}}$ The cell residence time of a cellular-only MS originated at double coverage area, $T_{c, d c a}^{c a}$, can be evaluated as $T_{\mathrm{c}, \mathrm{dca}}^{\mathrm{ca}}=T_{1}^{\mathrm{dca}}+T_{1}^{\mathrm{coa}}+\cdots+T_{N_{\mathrm{dca}}}^{\mathrm{dca}}+T_{N_{\mathrm{coa}}}^{\mathrm{coa}}$, where $N_{\mathrm{dca}}$ and $N_{\text {coa }}$ are the number of entrances of the double coverage area and the cellular-only area until the MS moves out the cell, respectively. On the other hand, the cell residence time of a cellular-only calls originated at the cellular-only area, $T_{\mathrm{c}, \mathrm{coa}}^{\mathrm{ca}}$ can be obtained as $T_{\mathrm{c}, \mathrm{coa}}^{\mathrm{ca}}=T_{1}^{\mathrm{coa}}+T_{1}^{\mathrm{dca}}+T_{2}^{\mathrm{coa}}+\cdots+T_{N_{\mathrm{dca}}}^{\mathrm{dca}}+T_{N_{\mathrm{coa}}}^{\mathrm{coa}}$.

We assume that the call duration $T_{v}$ follows an exponential distribution with mean $1 / \mu_{v}$ [7-9]. Since the call duration time and cell residence time are independent, the WLAN channel holding time of a WLAN-only call 
and a dual access call accepted in the WLAN can be obtained as $\min \left(T_{v}, T^{\mathrm{dca}}\right)$. Similarly, the channel holding times of cellular-only calls originated in the double coverage area and in the cellular-only area are given by min $\left(T_{v}, T_{\mathrm{c}, \mathrm{dca}}^{\mathrm{ca}}\right)$ and $\min \left(T_{v}, T_{\mathrm{c}, \mathrm{coa}}^{\mathrm{ca}}\right)$, respectively. The cellular channel holding times of dual access calls accepted by the cellular networks in the double coverage area and in the cellular-only area are obtained from $\min \left(T_{v}, T_{\mathrm{wc}, \mathrm{dca}}^{\mathrm{ca}}\right)$ and $\min \left(T_{v} T_{\mathrm{wc}, \text { coa }}^{\mathrm{ca}}\right)$, respectively.

\section{CAC with heterogeneous MSs}

In this section, we first introduce the motivation of the proposed call admission scheme. After that, the voice call capacities of WLANs and cellular networks are derived, and a CAC scheme with guard channels is proposed.

\subsection{Motivation}

WLAN-only MSs cannot retry to the cellular network even though their call requests to the WLAN are blocked while dual-interfaced MSs in the double coverage areas can retry the cellular network. Therefore, it is necessary to assign higher priority to call requests from WLANonly MSs to avoid unfairly higher blocking/dropping of the WLAN-only calls. Similar to the WLAN-only calls, cellular-only calls can access only the cellular networks, while dual-interfaced MSs have chances to access the WLAN in the double coverage area if their call requests to the cellular network are blocked. Dual-interfaced MSs try first the WLAN in the double coverage area to utilize the larger bandwidth of the WLAN. In addition, in the WLAN, vertical handoff calls have higher priority than new calls (e.g., WLAN-only or dual access calls) since the vertical handoff call dropping causes significant degradation in user satisfaction.

On the other hand, in the cellular network, dual access calls in the cellular-only area, cellular-only calls, horizontal handoff calls, and upward vertical handoff calls all compete for the same resource in the cellular network. We categorize these calls into (1) new calls including dual access calls blocked in the WLAN, (2) horizontal handoff, and (3) vertical handoff calls from the WLAN to the cellular networks. Dual access calls in the cellular-only area, cellular-only calls, and dual access calls blocked in the WLAN are eventually blocked if they are blocked in the cellular networks. Hence, these calls are classified into the same category. Upward vertical handoff calls are treated with the highest priority because of similar reasons with downward vertical handoff calls. Horizontal handoff calls have medium priorities since the dropping of these calls causes degradation in user satisfaction but horizontal handoffs do not need more signaling messages than vertical handoffs.

\subsection{Voice capacity of WLANs}

A voice call consists of uplink and downlink connections. When there are $N$ voice calls in the WLAN, the $N$ MSs send uplink voice traffic requests and all downlink traffic requests are processed at the AP. As reported in [10], in the distributed coordinated function, the collision probabilities of the MS and the AP, denoted as $p_{\mathrm{AP}}$ and $p_{\mathrm{MS}}$, respectively, are expressed as

$$
\begin{aligned}
& p_{\mathrm{AP}}=1-\left(1-\rho_{\mathrm{MS}} \tau_{\mathrm{MS}}\right)^{N} \\
& p_{\mathrm{MS}}=1-\left(1-\rho_{\mathrm{MS}} \tau_{\mathrm{MS}}\right)^{N-1}\left(1-\rho_{\mathrm{AP}} \tau_{\mathrm{AP}}\right) .
\end{aligned}
$$

where $\tau_{\mathrm{MS}}$ and $\tau_{\mathrm{AP}}$ are the transmission probabilities of the MS and the AP, respectively, and the $\rho_{\mathrm{AP}}$ and $\rho_{\mathrm{MS}}$ are the queue utilizations of the AP and MS, respectively. From Equations 1 and 2, the maximum number of voice calls when $\rho_{\mathrm{AP}}$ and $\rho_{\mathrm{MS}}$ are less than 1 (i.e., voice capacity $C^{\mathrm{w}}$ ) can be obtained.

\subsection{Voice capacity of the cellular network}

Since the uplink and the downlink are separate in time or frequency in cellular networks, the voice capacities of both the uplink and the downlink channels can be obtained individually. Then, we consider the voice capacity of cellular networks, $C^{\mathrm{c}}$, as the minimum value of the uplink and downlink voice capacities. The uplink voice capacity can be evaluated based on an uplink load factor [11], which can be expressed as

$$
\eta_{\mathrm{UL}}=\left(1+i_{\text {up }}\right) \cdot \sum_{j=1}^{N_{\text {up }}} \frac{1}{1+\frac{W}{\left(\frac{E_{b}}{N_{0}}\right)_{j} R_{j} v_{j}}}
$$

where $N_{\text {up }}, i_{\text {up }}, W,\left(\frac{E_{b}}{N_{0}}\right)_{j}, R_{j}$, and $v_{j}$ are the number of users in the own cell, the uplink other-to-own cell interference ratio, the chip rate, $\left(\frac{E_{b}}{N_{0}}\right)$ of the $j$ th user, the bit rate, and voice activity factor, respectively. Under the constraint of $\eta_{U L} \leq 1$, the uplink voice capacity can be determined.

The downlink capacity is limited by the transmission power of the base station $P_{\text {TOT }}$ and that can be expressed as [11]

$$
P_{\mathrm{TOT}}=\frac{P_{\mathrm{CCH}}+P_{N} \cdot \sum_{i=0}^{N_{\text {down }}} v_{i} \frac{\left(\frac{E_{b}}{N_{0}}\right)_{i}}{\frac{W}{R_{i}}} L_{i}}{1-\sum_{i=1}^{N_{\text {down }}} v_{i} \frac{\left(\frac{E_{b}}{N_{0}}\right)_{i}}{\frac{W}{R}}\left[\left(1-\alpha_{i}\right)+\bar{i}\right]}
$$


where $N_{\mathrm{down}}, \alpha_{i} P_{\mathrm{CCH}}, P_{N}, L_{i}$, and $\bar{i}$ are the number of downlink users in their own cell, the orthogonal factor of the cell, the power required for common channel, the noise power, the path loss, and the average downlink other-to-own cell interference ratio, respectively. For a given $P_{\text {TOT, }}$, the downlink voice capacity can be determined.

\subsection{CAC with guard channels}

Based on the voice capacities of the WLAN and the cellular networks, we describe the proposed CAC scheme with guard channels. To implement priorities assigned to WLAN-only calls and downward vertical handoff calls, we employ two guard channels parameters, $G_{\mathrm{w}}$ for the WLAN-only calls and $G_{\mathrm{vh}}^{\mathrm{w}}$ for the downward vertical handoff calls, i.e., as depicted in Figure 3, the downward vertical handoff calls can use the whole WLAN bandwidths, whereas the WLAN-only calls can be only admitted up to $N_{\mathrm{w}}^{\mathrm{w}}=C^{\mathrm{w}}-G_{\mathrm{vh}}$ and the dual access calls can be allowed up to $N_{\mathrm{wc}}^{\mathrm{w}}=C^{\mathrm{w}}-\left(G_{\mathrm{w}}+G_{\mathrm{vh}}^{\mathrm{w}}\right)$. Similarly, two guard channels $G_{\mathrm{hh}}$ for the horizontal handoff calls and $G_{\mathrm{vh}}^{\mathrm{c}}$ for the upward vertical handoff calls are used in the cellular network. As shown in Figure 4, the upward vertical handoff calls can be allowed up to the total capacity $C^{\mathrm{C}}$ whereas the horizontal handoff calls can be allowed up to $N_{h h}^{c}=C^{c}-G_{v h}^{c}$ and new calls can be admitted to $N_{n}^{c}=C^{c}-\left(G_{h h}+G_{v h}^{c}\right)$.

The proposed CAC scheme is summarized in Figure 5 ; if a call is requested in a cellular-only area, the proposed scheme operates as shown in Figure 5a. First, the proposed scheme determines if the incoming call request is a vertical handoff, a horizontal handoff, or a
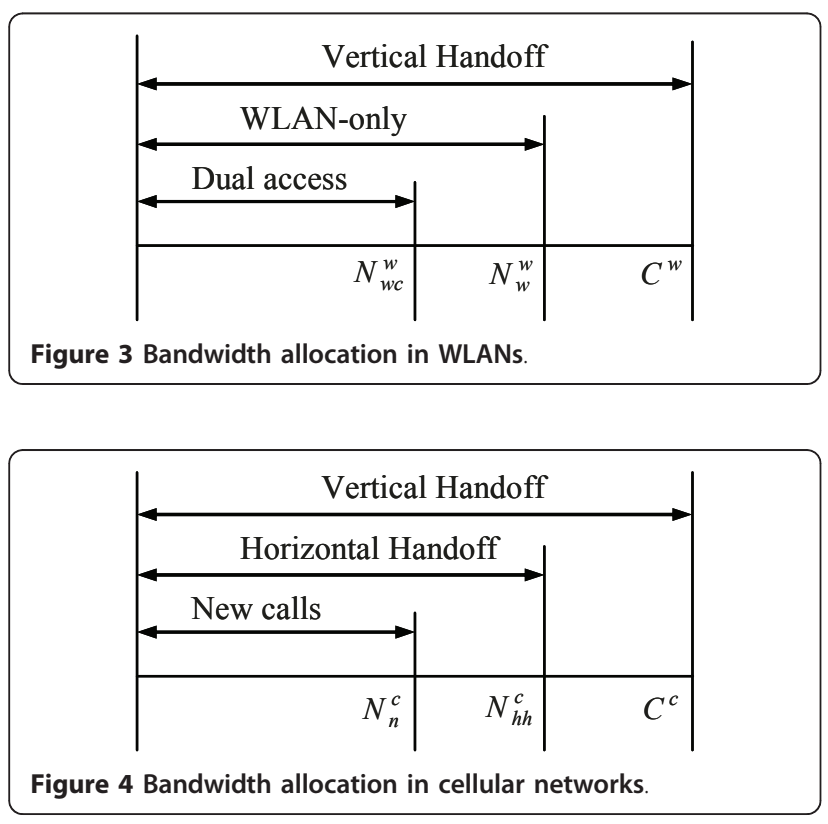

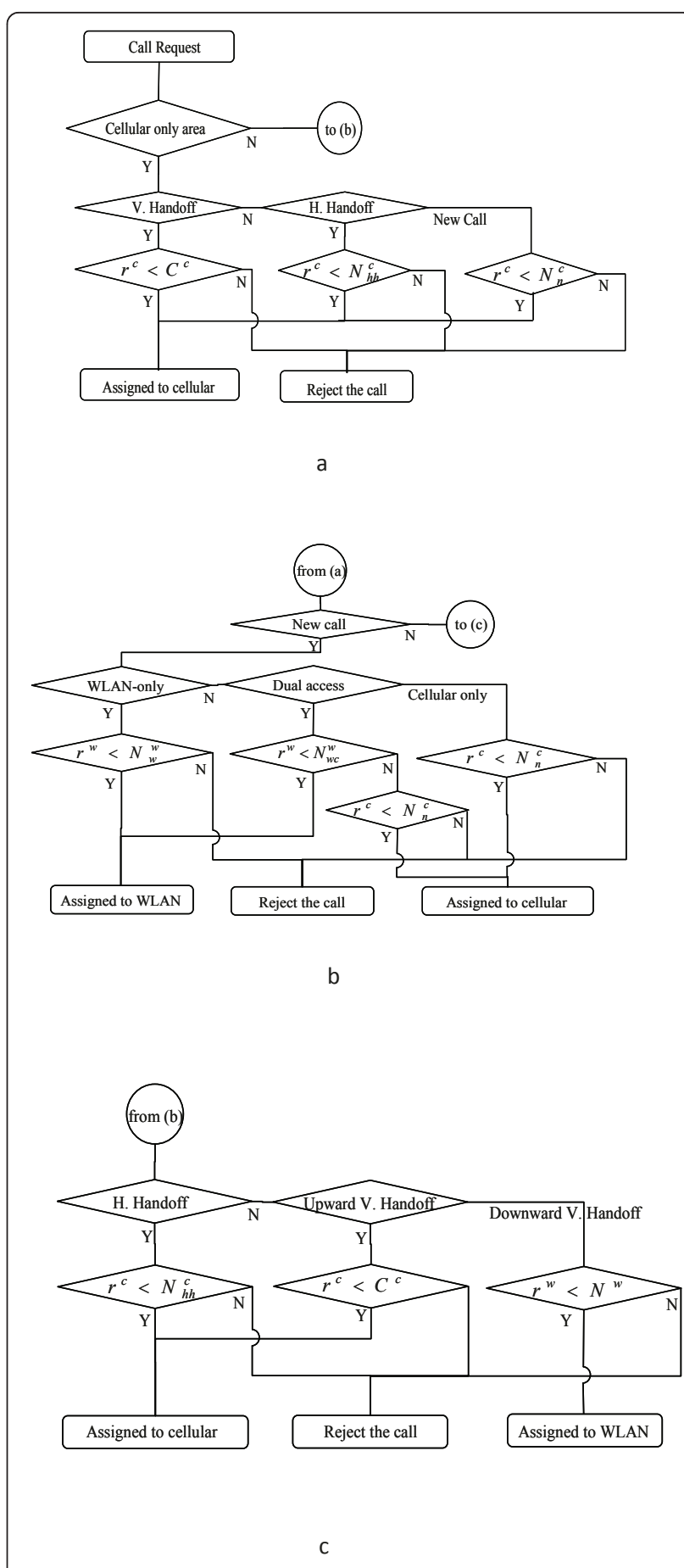

Figure 5 Flow diagrams (a) in a cellular only area (b) for new calls in a double coverage area and (c) for cellular only calls in a double coverage area.

new call. The new scheme uses three threshold values: $C^{c}$ for a vertical handoff, $N_{h h}^{c}$ for a horizontal handoff, and $N_{n}^{c}$ for a new call. The call request is admitted only when the number of used calls in a cellular network, $r^{c}$, is less than the corresponding threshold. On the other hand, if 
a call is requested in a double coverage area, the proposed scheme follows the procedure presented in Figure $5 \mathrm{~b}, \mathrm{c}$. The admission procedure for a new call is illustrated in Figure 5b. A WLAN-only call is admitted if the number of used calls in a WLAN, $r^{\mathrm{w}}$, is less than $N_{w}^{w}$, a dual access call is admitted to a WLAN if $r^{w}<N_{w}^{w}$ and to a cellular network if $r^{w} \geq N_{w}^{w}$ and $r^{c}<N_{n}^{c}$, and a cellular only call is admitted if $r^{c}<N_{n}^{c}$. The procedures for horizontal handoff, for upward vertical handoff, and for downward vertical handoff calls, are illustrated in Figure 5c. A horizontal handoff call is admitted to a cellular network if $r^{c}<N_{h h}^{c}$, an upward vertical handoff call is allowed to a cellular network if $r^{c}<C^{c}$, and a downward vertical handoff is admitted to a WLAN if $r^{w}<C^{w}$.

\section{Performance analysis}

For the purpose of performance evaluation, we analyze call dropping and blocking probabilities. To this end, we formulate the proposed scheme using Markov chains. The state of a WLAN is described as a row vector $\overrightarrow{n^{w}}=\left(n_{w c}^{w}, n_{w}^{w}\right)$ where $n_{w c}^{w}$ and $n_{w}^{w}$ are the numbers of dual access and WLAN-only calls in the WLAN, respectively. Similarly, the state of a cell can be described by a row vector $\overrightarrow{n^{c}}=\left(n_{w c}^{c}, n_{c}^{c}\right)$ where $n_{w c}^{c}$ and $n_{c}^{c}$ are the number dual access calls and cellular-only calls in the cell, respectively. In this section, arrival rates of new calls in each system, handoff rates, and departure rate are first described. Using these rates, Markov chains are constructed. Then, a method to solve these chains is introduced. Eventually, the guard band optimization scheme is described.

\subsection{Arrival rates in the WLAN}

To derive the arrival rates in the WLAN, we define an indicator function $I^{w}$ as

$$
I^{w}\left(\overline{n^{w}}, N^{w}\right)=\left\{\begin{array}{l}
1, \text { if } n_{w}^{w}+n_{w c}^{w}+1 \leq N^{w} \\
0, \text { otherwise }
\end{array}\right.
$$

where $N^{w}$ is a threshold to which a call is allowed up to, i.e., if a call can be admitted to the WLAN with the threshold, $I^{w}$ returns a " 1 ", otherwise, it returns a " 0 ". Therefore, the arrival rate of the dual access calls in the WLAN, $\lambda_{w c}^{w}$ is given by

$$
\lambda_{w c}^{w}=\lambda_{w c}^{d c a} I_{w c}^{d c a}+\lambda_{w c}^{c \rightarrow w} I_{w c}^{c \rightarrow w}
$$

where $I_{w c}^{d c a}$ and $I_{w c}^{c \rightarrow w}$ are the indicator functions for new calls in the dual access area and vertical handoff calls from the cell to the WLAN, respectively, i.e., $I_{w c}^{d c a}$ and $I_{w c}^{c \rightarrow w}$ represent as $I^{w}\left(\overline{n^{w}}, N_{w c}^{w}\right)$ and $I^{w}\left(\overline{n^{w}}, C^{w}\right)$, respectively.

On the other hand, the arrival rate of WLAN-only calls, $\lambda_{w}^{w}$, includes only newly arrived calls as

$$
\lambda_{w}^{w}=\lambda_{w}^{d c a} I_{w}^{d c a}
$$

where $I_{w}^{d c a}$ is the indicator function for the WLANonly calls and it equals to $I^{w}\left(\overline{n^{w}}, N_{w}^{w}\right)$.

Let $\pi\left(\overrightarrow{n^{w}}\right)$ be the steady-state probability of $\overrightarrow{n^{w}}$ in the WLAN. Then, dual access call blocking probability, $B_{w c}^{w, n}$, WLAN-only call blocking probability, $B_{w}^{w, n}$, and vertical handoff call dropping probability, $B_{w c}^{c \rightarrow w}$ can be obtained from

$$
\begin{aligned}
& B_{w c}^{w, n}=\sum_{\left|\overrightarrow{n^{w}}\right|=0}^{C^{w}} \pi\left(\overrightarrow{n^{w}}\right)\left(1-I^{w}\left(\overrightarrow{n^{w}}, N_{w c}^{w}\right)\right) \\
& B_{w}^{w, n}=\sum_{\left|\overrightarrow{n^{w}}\right|=0}^{C^{w}} \pi\left(\overrightarrow{n^{w}}\right)\left(1-I^{w}\left(\overrightarrow{n^{w}}, N_{w}^{w}\right)\right) \\
& B_{w c}^{c \rightarrow w}=\sum_{\left|\overrightarrow{n^{w}}\right|=0}^{C^{w}} \pi\left(\overrightarrow{n^{w}}\right)\left(1-I^{w}\left(\overrightarrow{n^{w}}, C^{w}\right)\right)
\end{aligned}
$$

\subsection{Arrival rates in the cellular network}

Similar to the indication function in the WLAN, the indicator function $I^{c}$ for the cellular network is defined as

$$
I^{c}\left(\overline{n^{c}}, N^{c}\right)=\left\{\begin{array}{l}
1, \text { if } n_{c}^{c}+n_{w c}^{c}+1 \leq N^{c} \\
0, \text { otherwise }
\end{array}\right.
$$

where $N^{c}$ is a threshold value up to which a call is allowed join the network.

Let $\lambda_{w c}^{c}$ be the arrival rate of the dual access call in the cell, which includes new calls, horizontal calls, and vertical handoff calls from the cell to join the WLAN. Then, $\lambda_{w c}^{c}$ is given by

$$
\lambda_{w c}^{c}=\left(\lambda_{w c}^{d c a} \times B_{w c}^{w, n}+\lambda_{w c}^{c o a}\right) I_{w c}^{c}+\lambda_{w c}^{c \rightarrow c} I_{w c}^{c \rightarrow c}+\lambda_{w c}^{w \rightarrow c} I_{w c}^{w \rightarrow c}
$$

where $I_{w c}^{c}, I_{w c}^{c \rightarrow c}$, and $I_{w c}^{w \rightarrow c}$ are the indicator functions for dual access calls, horizontal handoff calls, and vertical handoff calls, respectively. They are given by $I^{c}\left(\overrightarrow{n^{c}}, N_{h h}^{c}\right), I^{c}\left(\overrightarrow{n^{c}}, N_{h h}^{c}\right)$, and $I^{c}\left(\overrightarrow{n^{c}}, C^{c}\right)$, respectively.

On the other hand, the arrival rate of cellular-only calls, $\lambda_{c}^{c}$, includes new calls and horizontal handoff calls and is given by

$$
\lambda_{c}^{c}=\left(\lambda_{c}^{d c a}+\lambda_{c}^{c o a}\right) I_{c}^{c}+\lambda_{c}^{c \rightarrow c} I_{c}^{c \rightarrow c}
$$

where $I_{c}^{c}$ and $I_{c}^{c \rightarrow c}$ are the indicator functions for new cellular only calls and horizontal handoff calls and they are obtained as $I^{c}\left(\overrightarrow{n^{c}}, N_{n}^{c}\right)$ and $I^{c}\left(\overrightarrow{n^{c}}, N_{h h}^{c}\right)$, respectively.

Let $\pi\left(\overrightarrow{n^{c}}\right)$ be the steady state probability of $\overrightarrow{n^{c}}$ in the cell. The blocking probabilities of dual access calls and 
cellular-only calls, and the dropping probabilities of horizontal handoff calls of dual access MSs, horizontal handoff calls of cellular-only MSs, and vertical handoff calls are denoted as $B_{w c}^{c, n}, B_{c o}^{c, n}, B_{w c}^{c \rightarrow c}, B_{c}^{c \rightarrow c}$, and $B_{w c}^{w \rightarrow c}$, respectively, and they are given by

$$
\begin{aligned}
& B_{w c}^{c, n}=\sum_{\left|n^{c}\right|=0}^{C^{c}} \pi\left(\overrightarrow{n^{c}}\right)\left(1-I^{c}\left(\overrightarrow{n^{c}}, N_{n}^{c}\right)\right) \\
& B_{c}^{c, n}=\sum_{\left|\vec{n}^{c}\right|=0}^{C^{c}} \pi\left(\overrightarrow{n^{c}}\right)\left(1-I^{c}\left(\overrightarrow{n^{c}}, N_{n}^{c}\right)\right) \\
& B_{w c}^{c \rightarrow c}=\sum_{\left|\overrightarrow{n^{c}}\right|=0}^{C^{c}} \pi\left(\overrightarrow{n^{c}}\right)\left(1-I^{c}\left(\overrightarrow{n^{c}}, N_{h h}^{c}\right)\right) \\
& B_{c}^{c \rightarrow c}=\sum_{\left|\overrightarrow{n^{c}}\right|=0}^{C^{c}} \pi\left(\overrightarrow{n^{c}}\right)\left(1-I^{c}\left(\overrightarrow{n^{c}}, N_{h h}^{c}\right)\right) \\
& B_{w c}^{w \rightarrow c}=\sum_{\left|\overrightarrow{n^{c}}\right|=0}^{C^{c}} \pi\left(\overrightarrow{n^{c}}\right)\left(1-I^{c}\left(\overrightarrow{n^{c}}, C^{c}\right)\right)
\end{aligned}
$$

\subsection{Horizontal and vertical handoff rates}

Dual access calls accepted by a WLAN can make upward vertical handoffs which rates can be obtained through the multiplication of the transition probability from the double coverage to the cellular only area, $P^{w \rightarrow c}$, and the accepted rates by a WLAN. The upward vertical handoff can be expressed as

$$
\lambda_{w c}^{w \rightarrow c}=P^{w \rightarrow c} \times\left[\lambda_{w c}^{d c a} \times\left(1-B_{w c}^{w, n}\right)+\lambda_{w c}^{c \rightarrow w} \times\left(1-B_{w c}^{c \rightarrow w}\right)\right]
$$

where the first and the second terms on the righthand side mean the accepted new dual access and vertical handoff calls from a cellular to a WLAN, respectively, and $P^{w \rightarrow c}$ is obtained as $P^{w \rightarrow c}=P\left[T^{d c a}<T_{v}\right]=\eta^{d c a} /\left(\eta^{d c a}+\mu_{v}\right)$.

The calls admitted to a cellular network both in a double coverage and in a cellular-only area can make downward vertical handoffs to a WLAN. Their arrival rates can be obtained as

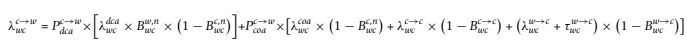

where the first term on the right-hand hand side means the vertical handoff rates of the dual access calls accepted by the cellular network in double coverage areas, the last terms on the right-hand side means the vertical handoff rates of dual access, horizontal handoff, and vertical handoff calls accepted to the cellular network in the cellular only area. Here, $P_{d c a}^{c \rightarrow w}$ and $P_{c o a}^{c \rightarrow w}$ are the vertical handoff probabilities of the calls accepted in the double coverage area and the cellular only area, respectively. The $P_{d c a}^{c \rightarrow w}$ can be evaluated as follows:

$$
P_{d c a}^{c \rightarrow w}=p^{c o a \rightarrow d c a} \times P\left[T_{v}>T_{w c, d c a}^{c a}\right]
$$

From [12], $P\left[T_{v}>T_{w c, d c a}^{c a}\right]$ can be computed from

$$
P\left[X>X_{0}+X_{1}+\cdots+X_{k}\right]=\frac{1}{2 \pi j} \int_{\sigma-j \infty}^{\sigma+j \infty} \frac{\prod_{i=0}^{k} f_{X_{i}}^{*}(s)}{s} f_{X}^{*}(-s) d s
$$

where $f_{X}^{*}, f_{X_{0}}^{*} f_{X_{1}}^{*}, \ldots, f_{X_{k}}^{*}$ are the Laplace transforms of random variables $X, X_{0}, X_{1}, \ldots, X_{\mathrm{k}}$, respectively.

Using Equation 17, $P_{d c a}^{c \rightarrow w}$ can be evaluated as

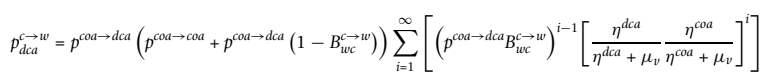

Similarly, $P_{c o a}^{c \rightarrow w}$ is given by

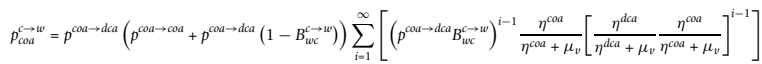

Both cellular-only calls accepted by a cellular network in a double coverage area and in a cellular-only area can make horizontal handoffs. The horizontal handoff rate can be obtained through multiplication of the transition probability to the neighboring cell and thus the accepted rates can be expressed as

$$
\lambda_{c}^{c \rightarrow c}=P_{c, d c a}^{c \rightarrow c} \times\left[\lambda_{c}^{c c a} \times\left(1-B_{c}^{c, n}\right)\right]+P_{c, c o a}^{c \rightarrow c} \times\left[\lambda_{c}^{c o a} \times\left(1-B_{c}^{c, n}\right)+\lambda_{c}^{c \rightarrow c} \times\left(1-B_{c}^{c \rightarrow c}\right)\right]
$$

where the first term on the right-hand side means the horizontal handoff rates of accepted cellular-only new calls in the double coverage area and the second term refers to those of new calls accepted in the cellular-only area and the horizontal handoff calls. The transition probability in a double coverage area to a neighboring cell, $P_{c, d c a}^{c \rightarrow c}$, and the transition probability in a cellular-only area to a neighboring cell, $P_{c, c o a}^{c \rightarrow c}$, can be obtained from

$$
\begin{aligned}
& P_{c, d c a}^{c \rightarrow c}=p^{c o a \rightarrow c o a} \times P\left[T_{v}>T_{c, d c a}^{c a}\right]=p^{c c a \rightarrow c o a} \sum_{i=1}^{\infty}\left[\left(p^{c o a \rightarrow d c a}\right)^{i-1}\left[\frac{\eta^{d c a}}{\eta^{d c a}+\mu_{v}} \frac{\eta^{c o a}}{\eta^{c o a}+\mu_{v}}\right]^{i}\right] \\
& P_{c, c o a}^{c \rightarrow c}=p^{c \omega a \rightarrow c o a} \times P\left[T_{v}>T_{c, c o a}^{c a}\right]=p^{c o u \rightarrow c o u} \sum_{i=1}^{\infty}\left[\left(p^{c o a \rightarrow d c a}\right)^{i-1} \frac{\eta^{c o a}}{\eta^{c o a}+\mu_{\nu}} \times\left[\frac{\eta^{d c a}}{\eta^{d c a}+\mu_{v}} \frac{\eta^{c o a}}{\eta^{c o d}+\mu_{\nu}}\right]^{i-1}\right]
\end{aligned}
$$

Similarly, the horizontal handoff of dual access calls can be obtained from

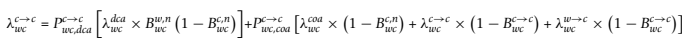

where $P_{w c, d c a}^{c \rightarrow c}$ and $P_{w c, c o a}^{c \rightarrow c}$ are computed as

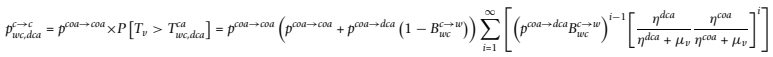

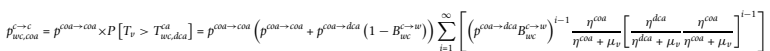




\subsection{Departure rates}

Departure rates can be obtained from the channel holding time which is the minimum time between the service time $T_{v}$ and the residence time $T_{r}$. If $T_{v}$ and $T_{r}$ are independent and $T_{v}$ follows an exponential distribution with mean $1 / \mu_{v}$, the expectation value of the channel holding time can be obtained as

$$
E\left[\min \left(T_{v}, T_{r}\right)\right]=E\left[T_{v}\right]-\int_{0}^{\infty} f_{T_{r}}(x) \frac{1}{\mu_{v}} e^{-\mu_{v} x} d x
$$

where $f_{\operatorname{Tr}}$ is the probability density function (pdf) of the residence time $T_{r}$. Equation 20 can be re-written with Laplace transformation as

$$
E\left[\min \left(T_{v}, T_{r}\right)\right]=E\left[T_{v}\right]-\frac{1}{\mu_{v}} f_{T_{r}}^{*}\left(\mu_{v}\right)
$$

where $f_{T_{r}}^{*}$ is the Laplace transformation of $f_{T_{r}}$.

Both dual access and WLAN-only calls accepted in the WLAN will release their channels when they move out the WLAN coverage or they are terminated. Therefore, by Equation 21, the departure rates of dual access and WLAN-only calls, denoted by $\mu_{w c}^{w}$ and $\mu_{w}^{w}$, respectively, can be expressed as $\mu_{w c}^{w}=\mu_{w}^{w}=\mu_{v}+\eta^{d c a}$.

The departure rates of the cellular-only calls accepted to the cell in the double coverage area, $\mu_{c}^{d c a}$ and in the cellular-only area, $\mu_{c}^{\text {coa }}$ are given by [7]

$$
\begin{aligned}
& \frac{1}{\mu_{c}^{d c a}}=\frac{1}{\mu_{v}}-\frac{1}{\mu_{v}} f_{T_{c, d d a}^{* a}}^{* a}\left(\mu_{v}\right)=\frac{1}{\mu_{v}}-\frac{1}{\mu_{v}} p^{c o a \rightarrow c o a} \sum_{i=1}^{\infty}\left[\left(p^{c o a \rightarrow d c a}\right)^{i-1}\left[\frac{\eta^{d c a}}{\eta^{d c a}+\mu_{v}} \frac{\eta^{c o a}}{\eta^{c o a}+\mu_{v}}\right]^{i}\right]
\end{aligned}
$$

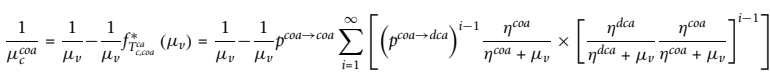

For the sake of tactical analysis, for cellular-only calls, we use the average departure rate, $\mu_{c}^{c}$, which is given by $\mu_{c}^{c}=r_{c}^{d c a} \mu_{c}^{d c a}+r_{c}^{c o a} \mu_{c}^{c o a}$, where $r_{c}^{d c a}$ and $r_{c}^{c o a}$ are the ratios of the cellular-only calls accepted in the double coverage area and in the cellular-only area. These ratios can be obtained from

$$
\begin{aligned}
& r_{c}^{d c a}=\frac{\lambda_{c}^{d c a} I_{c}^{c}}{\left(\lambda_{c}^{d c a}+\lambda_{c}^{c o a}\right) I_{c}^{\prime c}+\lambda_{c}^{c \rightarrow c} I_{c}^{\prime c \rightarrow c}} \\
& r_{c}^{c o a}=\frac{\lambda_{c}^{c o a} I_{c}^{\prime c}+\lambda_{c}^{c \rightarrow c} I_{c}^{c c \rightarrow c}}{\left(\lambda_{c}^{d c a}+\lambda_{c}^{c o a}\right) I_{c}^{\prime c}+\lambda_{c}^{c \rightarrow c} I_{c}^{\prime c \rightarrow c}}
\end{aligned}
$$

where $I_{c}^{\prime c}=I^{c}\left(\overrightarrow{n^{c}}, N_{n}^{c}+1\right)$ and $I_{c}^{\prime c \rightarrow c}=I^{c}\left(\overrightarrow{n^{c}}, N_{h h}^{c}+1\right)$.

The departure rates of dual access calls in the double coverage area, $\mu_{w c}^{d c a}$, and in the cellular-only area, $\mu_{w c}^{c o a}$ can be obtained from

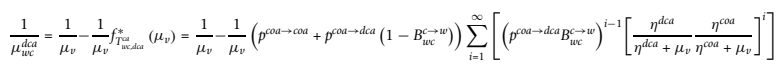

and

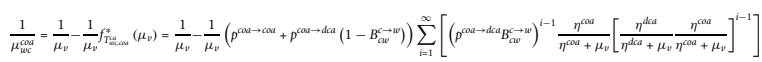

Similar to the cellular-only calls, the average departure rate, $\mu_{w c}^{c}$, for dual access calls is used and it is computed as $\mu_{w c}^{c}=r_{w c}^{d c a} \mu_{w c}^{d c a}+r_{w c}^{c o a} \mu_{w c}^{c o a}$, where $r_{w c}^{d c a}$ and $r_{w c}^{c o a}$ are the ratios of dual access accepted in the double coverage area and in the cellular-only area. These ratios are obtained from

$$
\begin{gathered}
r_{w c}^{d c a}=\frac{\lambda_{w c}^{d c a} B_{w c}^{w, n} I_{w c}^{\prime c}}{\lambda_{w c}^{\prime c}} \\
r_{i w c}^{c o a}=\frac{\lambda_{w c}^{c o a} I_{w c}^{c}+\lambda_{w c}^{c \rightarrow c} I_{w c}^{c \rightarrow c}+\left(\lambda_{w c}^{w \rightarrow c}+\tau_{w c}^{w \rightarrow c}\right) \lambda_{w c}^{w \rightarrow c} I_{w c}^{w \rightarrow c}}{\lambda_{w c}^{\prime c}}
\end{gathered}
$$
and

where

$$
\begin{aligned}
& I_{w c}^{c}=I^{c}\left(\overrightarrow{n^{c}}, N_{h h}^{c}+1\right), I_{w c}^{c}=I^{c}\left(\overrightarrow{n^{c}}, N_{n}^{c}+1\right),- \\
& I_{w c}^{\prime c}=I^{c}\left(\overrightarrow{n^{c}}, N_{h h}^{c}+1\right), \text { and } I_{w c}^{c}=I^{c}\left(\overrightarrow{n^{c}}, C^{c}+1\right) .
\end{aligned}
$$

\subsection{State diagrams for WLANs and cellular networks}

With arrival and termination rates mentioned above, the state diagrams in WLANs and cellular networks are illustrated in Figures 6 and 7, respectively. The state-dependent transition rates in Figures 6 and 7 are given by

$$
\left(n_{w^{\prime}}^{w} n_{w c}^{w}\right) \rightarrow\left(n_{w}^{w}, n_{w c}^{w}+1\right)
$$

(1) $\lambda_{w c}^{d c a}+\lambda_{w c}^{c \rightarrow w}$, if $n_{w}^{w}+{ }_{w c}^{w} \leq N_{w c}^{w}$

(2) $\lambda_{w c}^{c \rightarrow w}$, if $n_{w}^{w}+w c \leq C^{w}$

$$
\left(n_{w}^{w}, n_{w c}^{w}\right) \rightarrow\left(n_{w}^{w}+1, n_{w c}^{w}\right)
$$

(3) $\lambda_{w c}^{c \rightarrow w}$, if $n_{w^{\prime}}^{w}+{ }_{w c}^{w} \leq N_{w}^{w}$

$$
\left(n_{w}^{w}, n_{w c}^{w}\right) \rightarrow\left(n_{w}^{w}, n_{w c}^{w}-1\right)
$$

(4) $n_{w c}^{n} \mu_{w c}^{w}$, if $1 \leq n_{w c}^{w} \leq C^{w}$

$$
\left(n_{w}^{w}, n_{w c}^{w}\right) \rightarrow\left(n_{w}^{w}-1, n_{w c}^{w}\right)
$$

(5) $n_{w}^{n} \mu_{w}^{w}$, if $1 \leq n_{w}^{w} \leq N_{w}^{w}$

$$
\left(n_{c^{\prime}}^{c}, n_{w c}^{c}\right) \rightarrow\left(n_{c^{\prime}}^{c}, n_{w c}^{c}+1\right)
$$

(6) $\lambda_{w c}^{n}+\lambda_{w c}^{c \rightarrow c}+\lambda_{w c}^{w \rightarrow c}$, if $n_{c}^{c}+{ }_{w c}^{c} \leq N_{n}^{c}$

(7) $\lambda_{w c}^{c \rightarrow c}+\lambda_{w c}^{w \rightarrow c}$, if $n_{c}^{c}+{ }_{w c}^{c} \leq N_{h h}^{c}$

(8) $\lambda_{w c}^{w \rightarrow c}$, if $n_{c}^{c}+{ }_{w c}^{c} \leq C^{c}$

$$
\left(n_{c}^{c}, n_{w c}^{c}\right) \rightarrow\left(n_{c}^{c}+1, n_{w c}^{c}\right)
$$

(9) $\lambda_{c}^{n}+\lambda_{c}^{c \rightarrow c}$, if $n_{c}^{c}+{ }_{w c}^{c} \leq N_{n}^{c}$

(10) $\lambda_{c}^{c \rightarrow c}$, if $n_{c}^{c}+{ }_{w c}^{c} \leq N_{h h}^{c}$

$$
\left(n_{c^{\prime}}^{c} n_{w c}^{c}\right) \rightarrow\left(n_{c^{\prime}}^{c} n_{w c}^{c}-1\right)
$$




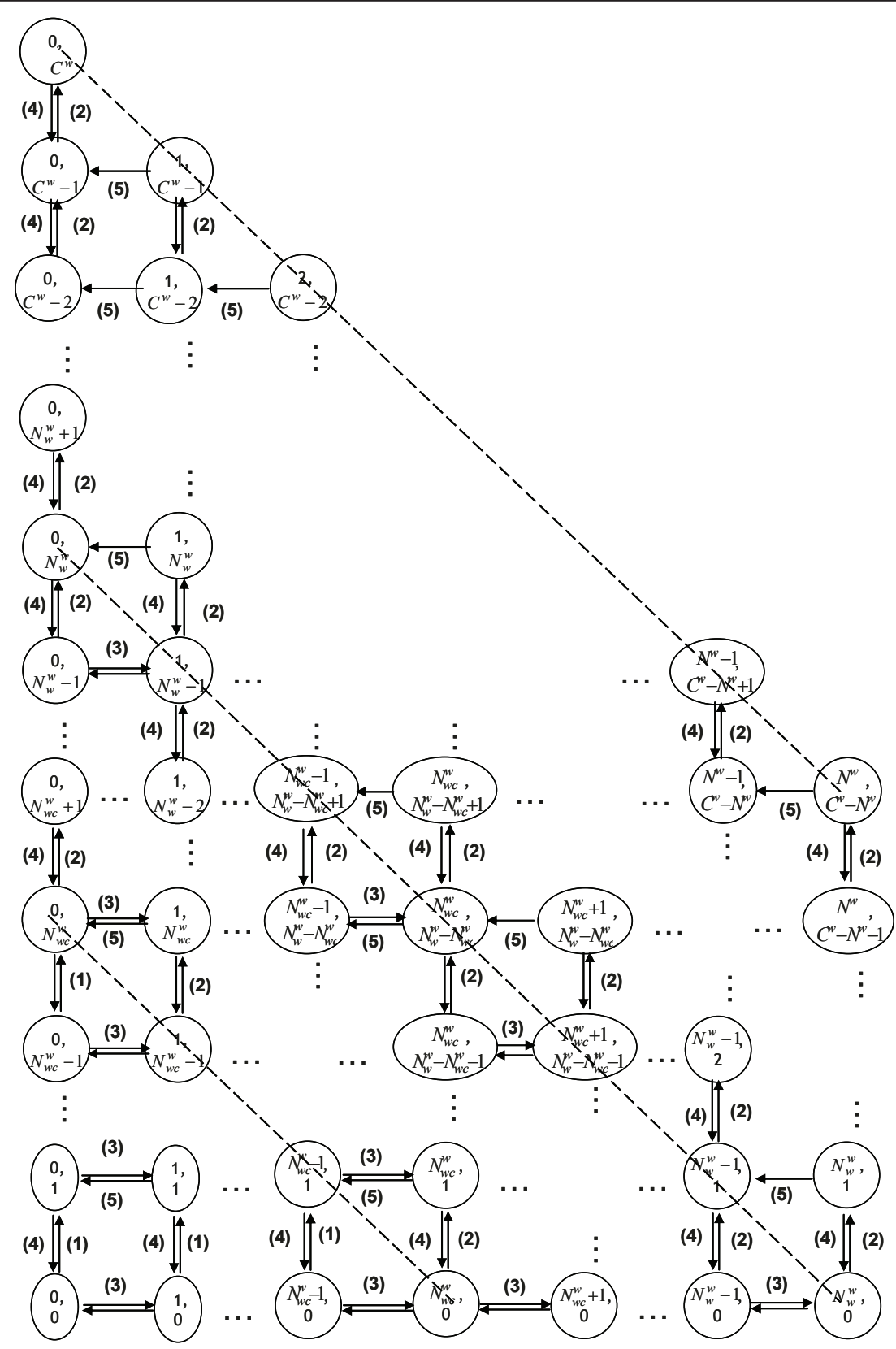

Figure 6 Markov chain of the proposed scheme in WLANs.

(11) $n_{w c}^{c} \mu_{w c}^{c}$, if $1 \leq n_{c}^{c}+_{w c}^{c} \leq C^{c}$

$$
\left(n_{c^{\prime}}^{c} n_{w c}^{c}\right) \rightarrow\left(n_{c}^{c}-1, n_{w c}^{c}\right)
$$

(12) $n_{c}^{c} \mu_{c}^{c}$, if $1 \leq n_{c}^{c}{ }_{w c}^{c} \leq N_{h h}^{c}$

\subsection{Iterative methods for computing steady-state probabilities}

After obtaining the arrival and the departure rates, we need to compute the steady-states $\pi\left(\overrightarrow{n^{w}}\right)$ and $\pi\left(\overrightarrow{n^{c}}\right)$. However, the states of the WLAN and the cellular networks are not independent due to the retrials of dual access calls blocked in WLANs and vertical handoffs. Hence, we use an iterative approach in which one-step results for one network are used for inputs for obtaining the steady states in another network [9]. The detailed algorithms are as follows:

1: Set initial $\varepsilon$

2: Set initial values as follows

All blocking probabilities in Equations 4-7 and $10-14=0$,

All handoff rates in Equations 15, 16, 18, $19=0$ 


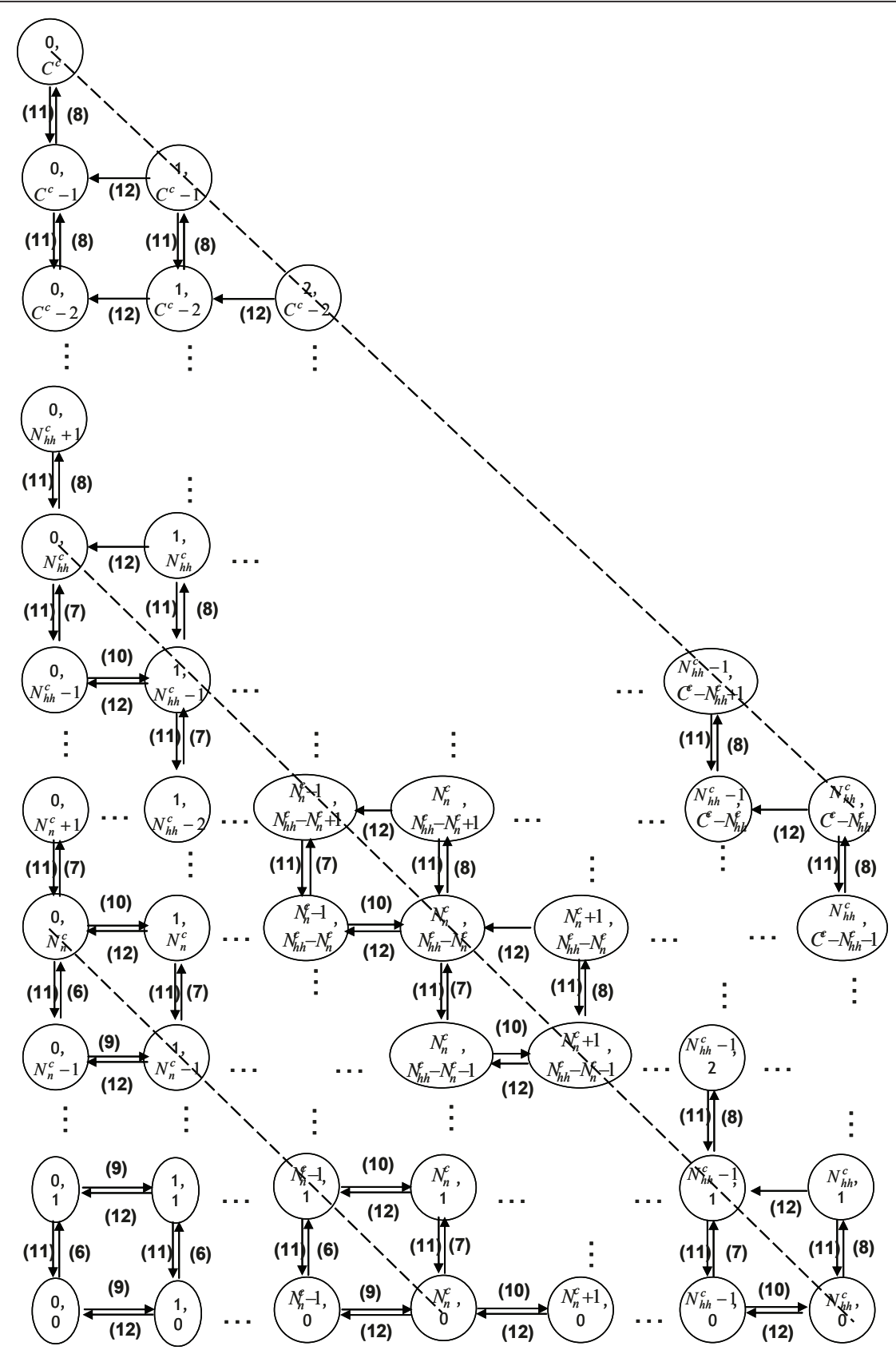

Figure 7 Markov chain of the proposed scheme in cellular networks.

3: While $\sum \mid$ old $B-$ new $B \mid>\varepsilon$

4: In the WLAN

(1) Compute the arrival rates in Equations 3 and 4

(2) Compute all the steady-state probability, $\pi\left(\overrightarrow{n^{w}}\right)$, by solving global balance equations through

$$
\pi\left(\overrightarrow{n^{w}}\right) Q^{W}=0 \text { and } \pi\left(\overrightarrow{n^{w}}\right) \cdot \vec{e}=1
$$

where $Q^{w}$ is the generator matrix of the WLAN.

(3) Obtain new blocking probabilities
(4) Update blocking probabilities

5: In the cellular network

(1) Compute the arrival rates in Equations 8 and 9

(2) Compute all the state probabilities by solving global balance equation using equations through

$$
\pi\left(\overrightarrow{n^{c}}\right) Q^{c}=0 \text { and } \pi \overrightarrow{\left(n^{c}\right)} \cdot \vec{e}=1
$$

where $Q^{c}$ is the generator matrix of the cellular system 
(3) Obtain the new blocking probabilities

(4) Update the blocking probabilities

6: End

\subsection{Optimization problem}

In the proposed CAC scheme, guard bands play key roles in the performance. Therefore, to obtain the guard bands for the optimal performance, we formulate the optimization problem to minimize the blocking probabilities, which is equivalent to maximizing the accepted calls. The linear cost function for the optimization problem, $Q$, can be expressed as

$$
Q=\alpha_{n e w} C_{n e w}+\alpha_{h h} C_{h h}+\alpha_{v h} C_{v h}
$$

where $\alpha_{n e w}, \alpha_{h h}$, and $\alpha_{v h}$ are weighting factors for blocked new, horizontal handoff, and vertical handoff calls, respectively, $Q_{n e w}, Q_{h h}$, and $Q_{v h}$ are the cost function for accepted new, horizontal handoff, and vertical handoff calls and they are given by

$$
\begin{aligned}
& Q_{n e w}=\lambda_{w}^{d c a} B_{w}^{w, n}+\lambda_{w c}^{d c a} B_{w c}^{w, n} B_{w c}^{c, n}+\lambda_{w c}^{c o a} B_{w c}^{c, n}+\lambda_{c}^{d c a} B_{c}^{c, n}+\lambda_{c}^{c o a} B_{c}^{c, n} \\
& Q_{h h}=\lambda_{c}^{c \rightarrow c} B_{w c}^{c \rightarrow c}+\lambda_{w c}^{c \rightarrow c} B_{w c}^{c \rightarrow c} \\
& Q_{v h}=\lambda_{w c}^{w \rightarrow c} B_{w c}^{w \rightarrow c}+\lambda_{w c}^{c \rightarrow w} B_{w c}^{c \rightarrow w}
\end{aligned}
$$

Hence, the optimization problem in the proposed scheme can be expressed as

$$
\underset{N_{w c}^{w}, N_{w}^{w}, N_{n}^{c}, N_{h h}^{c}}{\operatorname{minimize}} Q
$$

The above optimization problem can be solved by means of exhaustive search or meta-heuristic algorithms $[9,13]$.

\section{Simulation results}

In this section, we present analytical and simulation results of the proposed scheme compared with the WLAN-first scheme [7] that has guard bands for horizontal handoff and vertical handoff calls. For the simulations, Matlab 7.0 is used. It is notes that the WLAN-first scheme does not consider single-interfaced MSs.

To determine the voice capacity of the WLAN, we assume G.711 codec with a sampling rate of $20 \mathrm{~ms}$, which generates voice packets of 80 bytes. Then, based on the description in Section 3.2, the voice capacity is given by 11 calls. On the other hand, for the voice capacity in cellular networks, the power of the base station $P_{T O T}$ and the power of the common channel $P_{C C H}$ are set to 20 and $3.6 \mathrm{~W}$, respectively. In addition, the chip rate, $\mathrm{W}$, is 3.84 Mcps and the voice data rate is assumed as $12.2 \mathrm{kbps}$ with the adaptive multi-rate (AMR) codec.
The values of $\left(\frac{E_{b}}{N_{0}}\right)$ for uplink and downlink are set to 5.9 and $7.4 \mathrm{~dB}$, respectively. The voice activity is $50 \%$, which leads the physical layer activity factor of $67 \%$ in uplink and 58\% in downlink. The downlink orthogonality is 0.5 and other-cell to own-cell interference ratio is 0.65 [11]. Based on these parameters, the voice capacity of a cell, $C^{\mathrm{c}}$, is set to 70. Based on [7], the mobilityrelated parameters have the following values: $1 / \eta^{c o}=10$ $\min , 1 / \eta^{d c}=14 \mathrm{~min}, p^{\text {coa } \rightarrow \mathrm{dca}}=0.24$, and $p^{\text {coa } \rightarrow \text { coa }}=$ 0.76 .

\subsection{Proposed scheme versus WLAN-first scheme}

We first analyze the call blocking probabilities when $N_{w c}^{w}$ $=6, N_{w}^{w}=9, N_{n}^{c}=60$, and $N_{h h}^{c}=65$. It is noted that the blocking probability of WLAN-only calls can be computed by considering only blocking in the WLAN. On the contrary, dual access calls are blocked when they are blocked in both the WLAN and the cellular network.

From Figure 8, it can be seen that the blocking probability of the WLAN-only calls is unfairly higher than that of dual access calls. Moreover, with the increase of the arrival rates of the dual access calls, the blocking probability of the WLAN-only calls significantly increases. This is because the WLAN-only and dual access calls are treated with the same priority in the WLAN-first scheme even though the dual access calls have additional chances to access cellular networks. On the other hand, the proposed scheme gives a higher priority to WLAN-only calls using the threshold, $N_{w c}^{w}$, which dual access calls are allowed up to. Hence, in the proposed scheme, the WLAN-only calls are protected from the increase in the blocking probabilities in case of the arrival rates of dual access calls exceed a certain threshold.

From Figure 9, it can be shown that WLAN-only calls exhibit low blocking probabilities in the proposed scheme if the arrival rate of the WLAN-only calls is low. This is because WLAN-only calls are protected from dual access calls by means of the guard bandwidth with thresholds, $N_{w}^{w}=6$ and $N_{w}^{w}=9$.

Figure 10 illustrates the total number of blocked calls in the WLAN-first and proposed schemes. It can be found that the proposed scheme shows a lower number of blocked calls (or better utilization) than the WLANfirst scheme even though the guard bands are not optimized.

\subsection{Guard band optimization}

We use 1,7 , and 10 for the values of the weighting factors, $\alpha_{n}, \alpha_{h h}$, and $\alpha_{v h}$ for the optimization problems, respectively. The weighting factor for vertical handoff calls with the highest priority is set to be 10 and that 


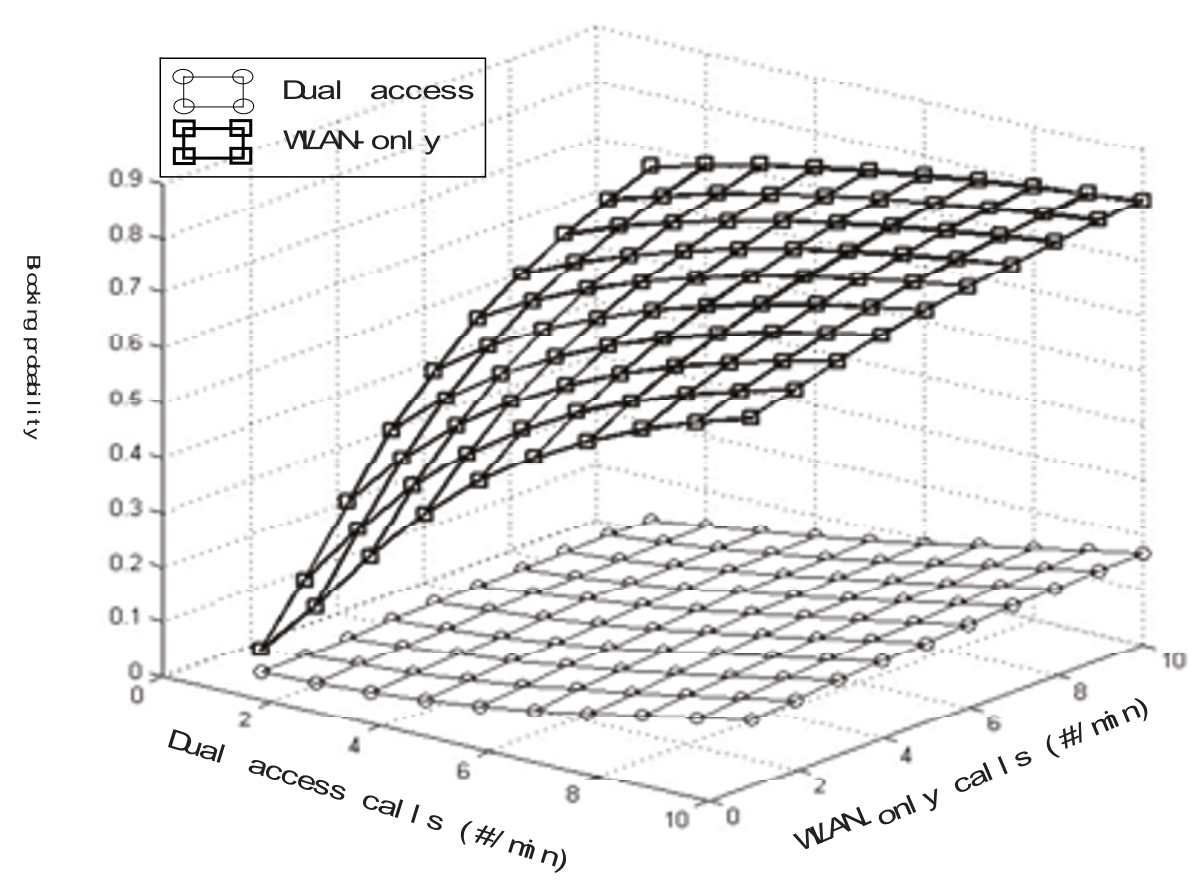

Figure 8 Blocking probability in the WLAN-first scheme.

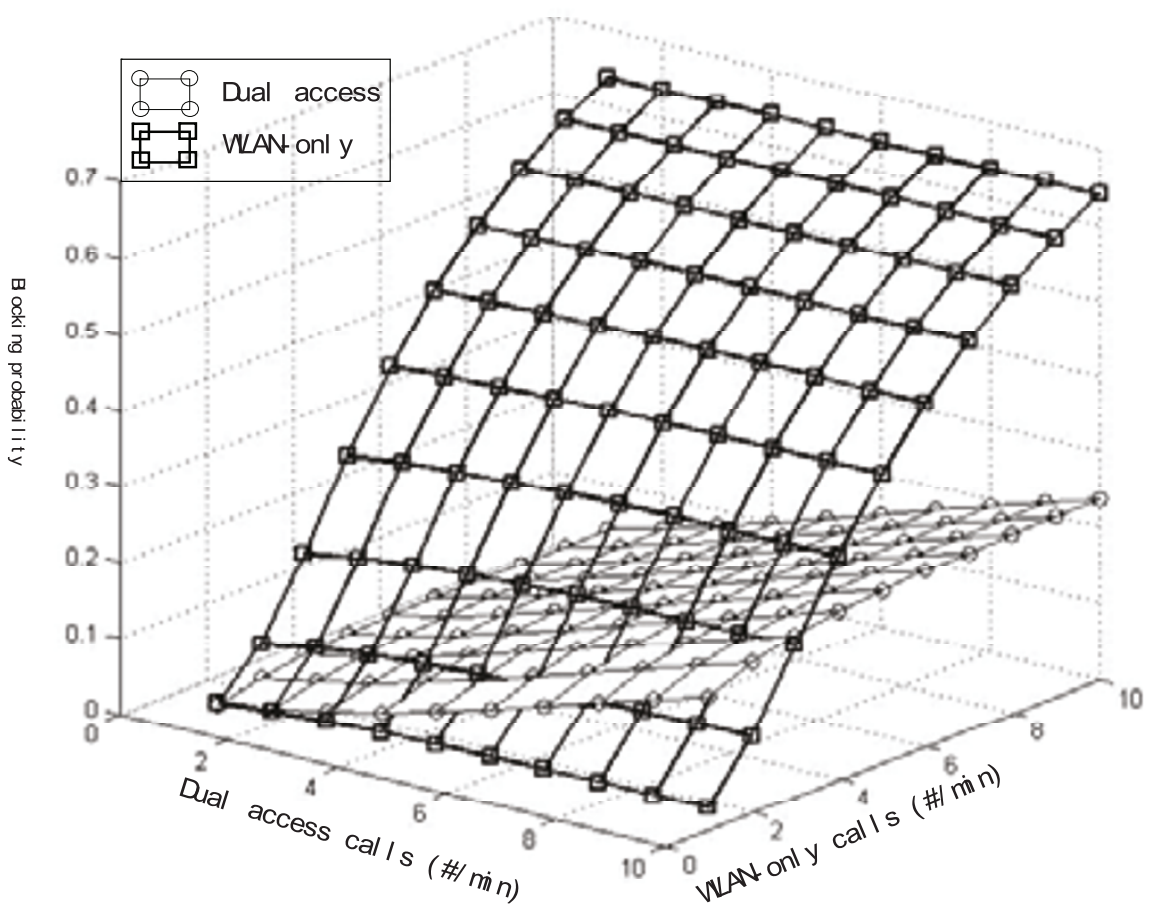

Figure 9 Blocking probability in the proposed scheme. 


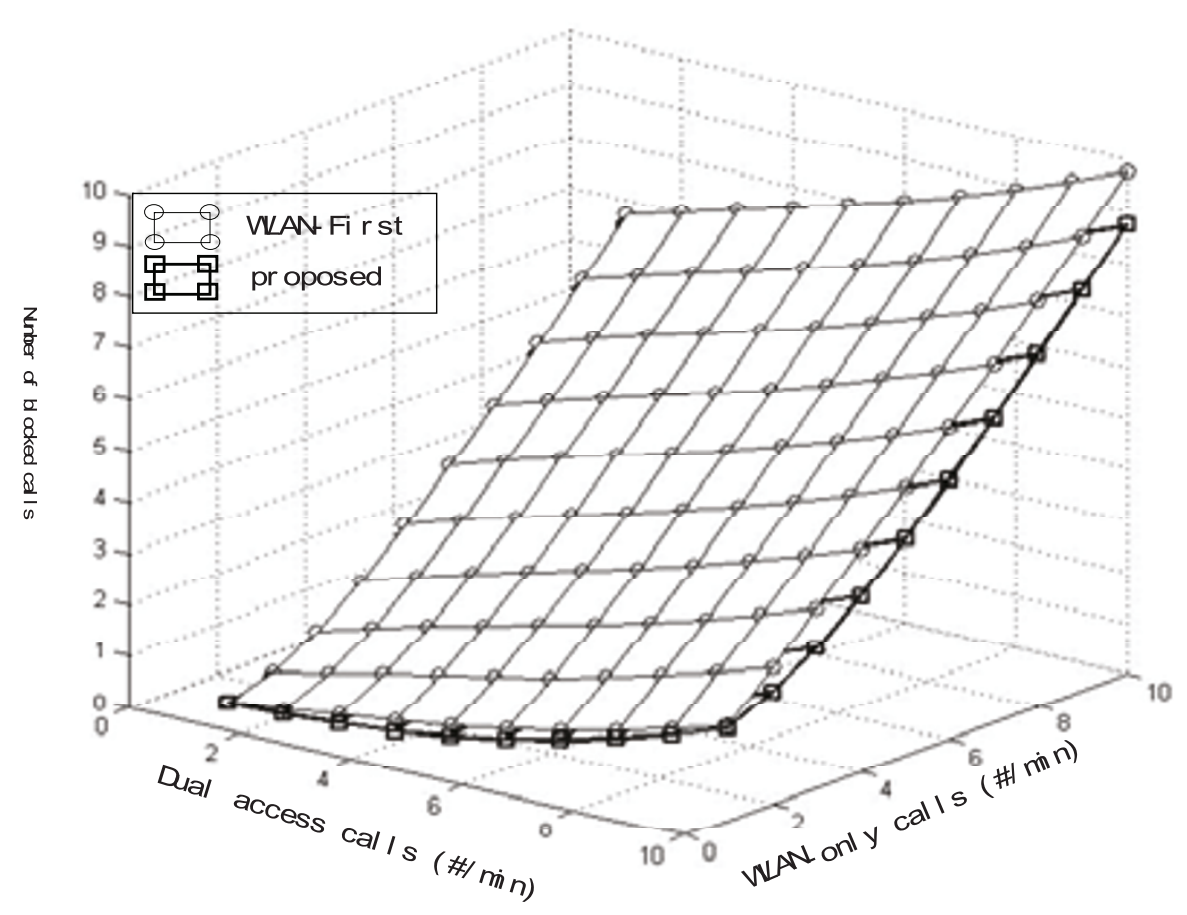

Figure 10 Numbers of blocked calls.

for horizontal handoff calls is set to 7 , since these calls have lower priority than vertical handoff calls.

We first consider medium traffic load in the cellular network with $\lambda_{c}^{d c a}=5, \lambda_{w c}^{c o a}=5$, and $\lambda_{c}^{c o a}=5$ and evaluate the proposed scheme in the double coverage area. Results for the optimization problem with this case are illustrated in Figure 11 and the corresponding thresholds $N_{w c}^{w}$ and $N_{w}^{w}$ are given in Figure 12. It can be seen that, for a given arrival rate of WLAN-only calls, the difference in the cost values between the proposed scheme and the WLAN-first scheme increases as the dual call arrival rate increases. When the arrival rate of the WLAN-only calls is low, the proposed scheme increases $N_{w c}^{w}$ to utilize more WLAN bandwidths. On the other hand, when the arrival rate of WLAN-only calls is high, the proposed scheme sets $N_{w c}^{w}$ to a low value, which makes more dual access calls try to cellular networks, which results in reduced blocking probabilities. Figure 11 also shows that the effectiveness of the proposed scheme against the WLAN-first scheme slightly increases as the arrival rate of the WLAN-only calls increases for a given arrival rate of dual access calls.

Figure 13 highlights the gain of the proposed scheme against the WLAN-first scheme and distributed access scheme [8]. As shown in Figure 13, at low arrival rates of WLAN-only calls, the distributed access scheme shows better performance than the WLAN-First scheme since dual access calls are distributed to WLAN and cellular networks. On the other hand, the distributed access scheme and the WLAN-first scheme exhibit comparable performance as the arrival rate of WLAN-only calls increases since WLAN-only calls are not fairly treated. However, it can found that the proposed scheme can reduce the blocking cost by treating WLAN-only calls in a better way. Figure 14 shows the blocking cost as a function of residence time in the double coverage area. From Figure 14, it can be seen that the proposed scheme has better performance even in high mobility conditions than the WLAN-first scheme and the distributed access scheme.

To verify numerical analysis, we perform even-driven simulation using MATLAB with the same arrival and departure rates as used for numerical analysis. In Figure 15 , analytical and simulation results for the cost value $Q$ and thresholds $N_{w c}^{w}$ and $N_{w}^{w}$ are shown together for the comparison as function of dual access call arrival rate with $\lambda_{w}^{d c a}=1, \lambda_{c}^{d c a}=5, \lambda_{w c}^{c o a}=5$, and $\lambda_{c}^{c o a}=5$. As shown in Figure 15, analytical and simulation results are well matched.

In the second case, we consider heavy traffic loads in the cellular network with $\lambda_{c}^{d c a}=10, \lambda_{w c}^{c o a}=10, \lambda_{c}^{c o a}=10$. From Figure 16, it can be found that the proposed scheme has comparable performance to the WLAN-first scheme. When the traffic load in the cellular network, dual access calls are more likely to be blocked by the cellular network in the double coverage area, and these 


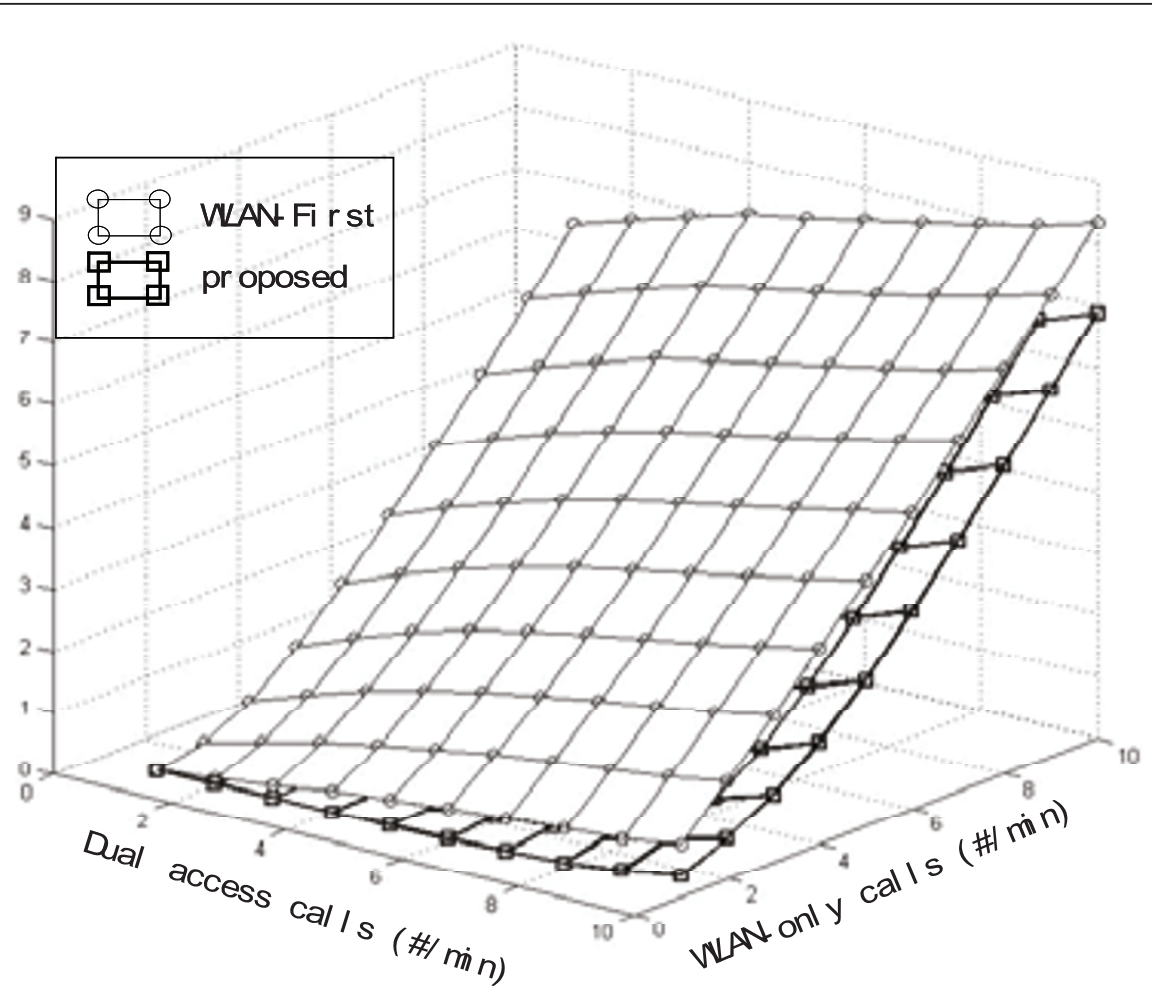

Figure 11 Results of guard band optimization with $\lambda_{c}^{d c a}=5, \lambda_{w c}^{c o a}=5$, and $\lambda_{c}^{c o a}=5$.

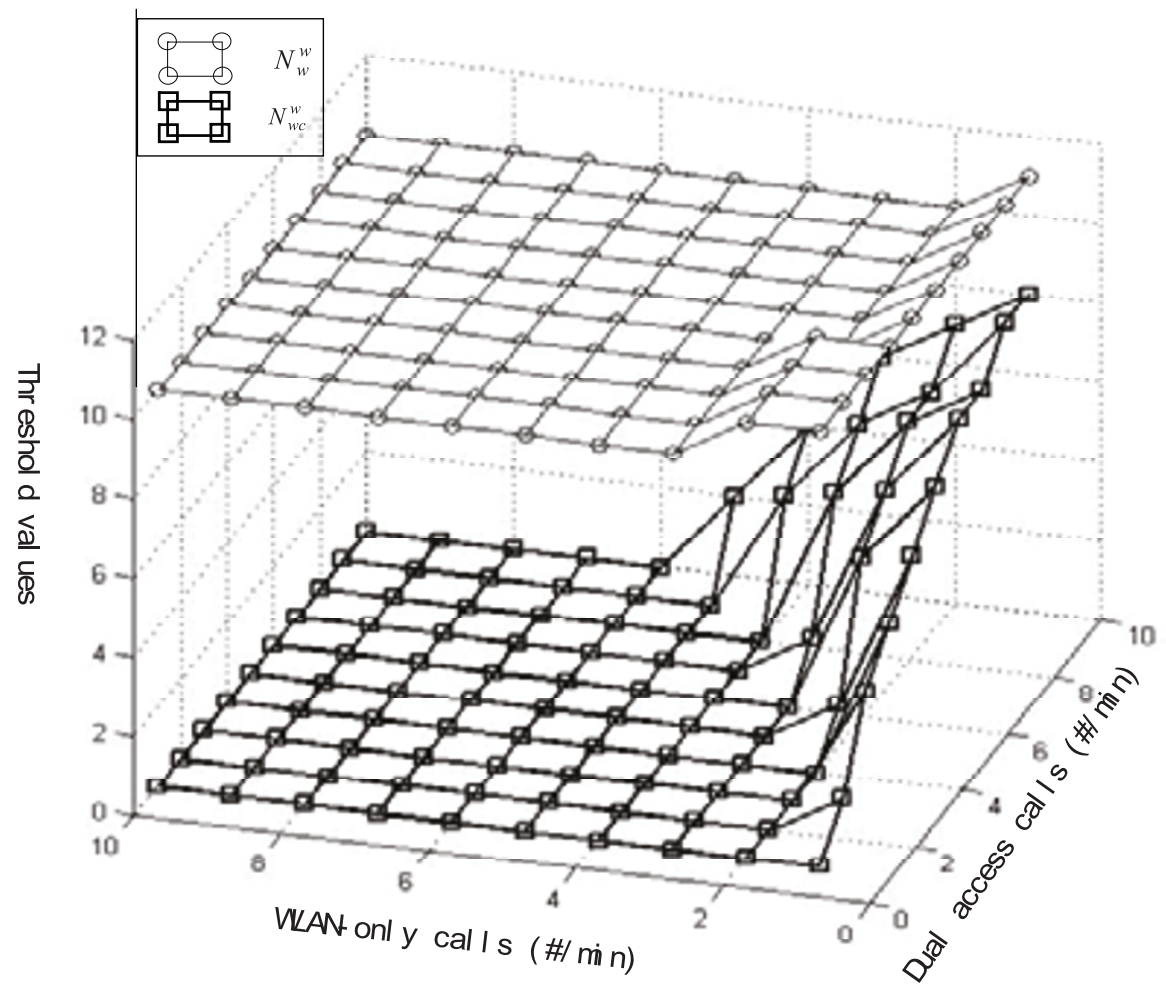

Figure 12 Threshold values of guard band optimization with $\lambda_{c}^{d c a}=5, \lambda_{w c}^{c o a}=5$, and $\lambda_{c}^{c o a}=5$. 


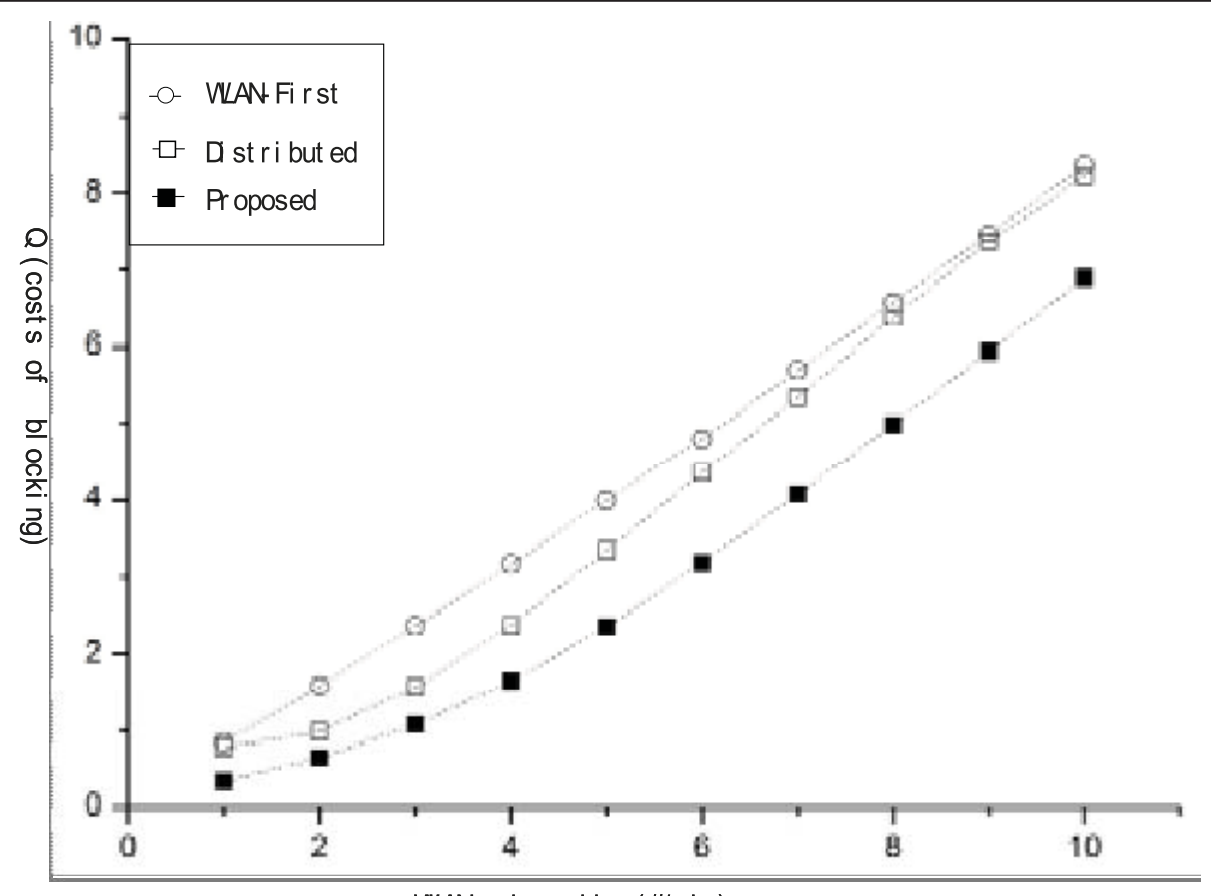

WAN onl y cal I s (\#/ min)

Figure 13 Proposed vs. WLAN-first versus distributed access $\left(\lambda_{c}^{d c a}=10, \lambda_{c}^{d c a}=5 \lambda_{w c}^{c o a}=5\right.$, and $\left.\lambda_{c}^{c o a}=5\right)$.

calls will be redirected to the WLAN. Since this operation is similar both in the proposed scheme and the WLAN-first scheme, they show comparable performance under this situation.

\section{Conclusions}

In this article, we propose a voice CAC scheme that considers heterogeneous MSs with WLAN-only, cellular only, and both interfaces in cellular/WLAN-integrated

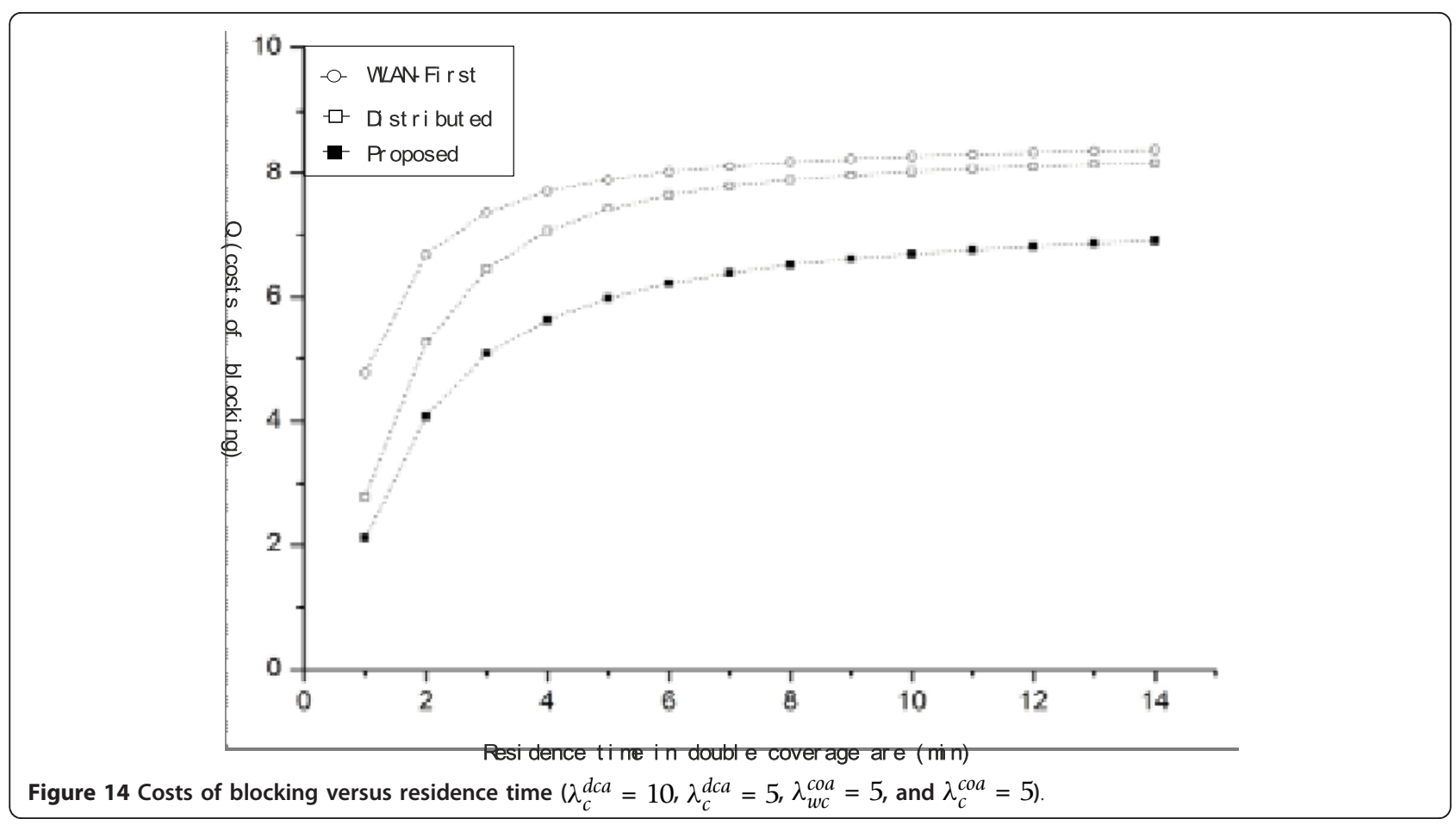




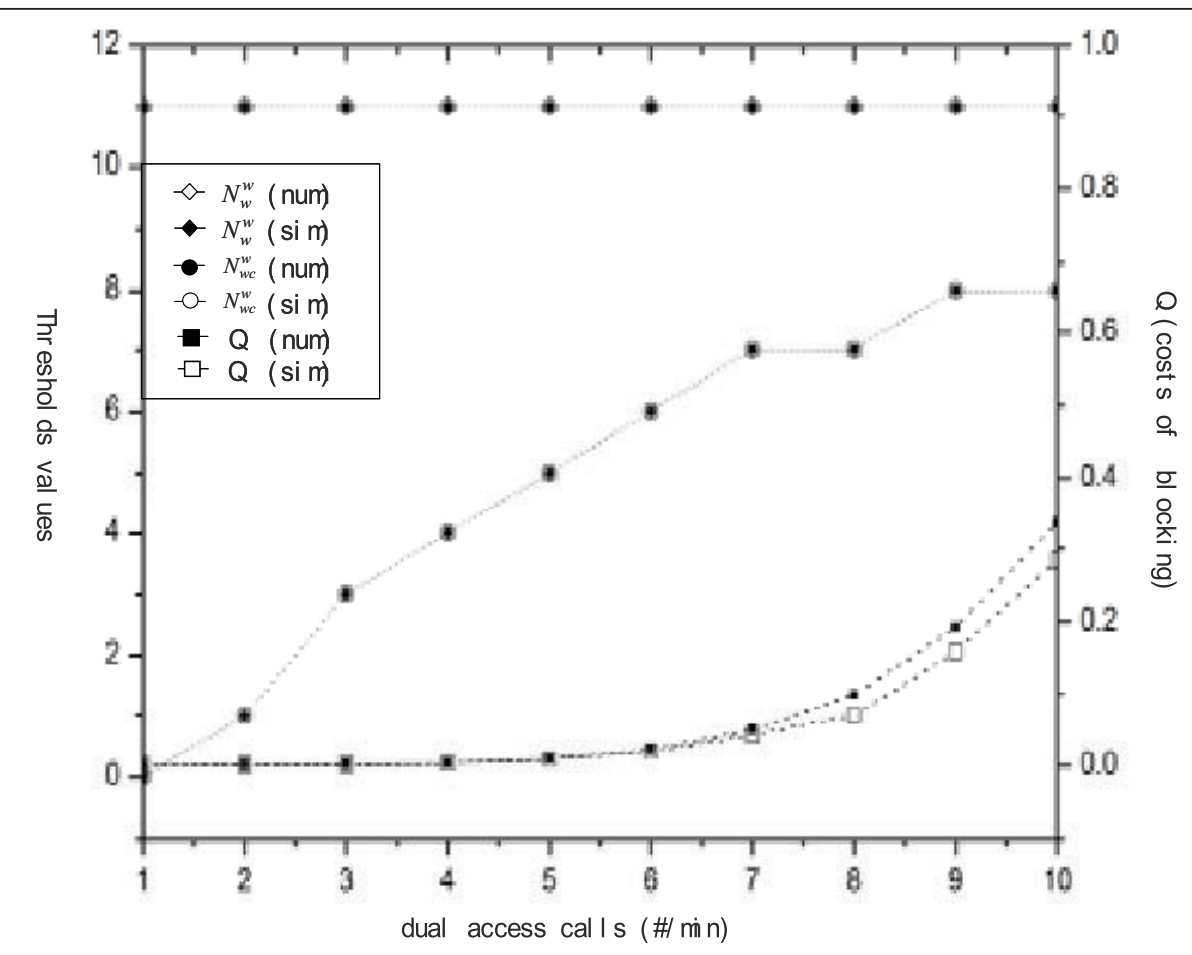

Figure 15 Analytical and simulation results with $\lambda_{w}^{d c a}=1, \lambda_{c}^{d c a}=5, \lambda_{w c}^{c o a}=5$, and $\lambda_{c}^{c o a}=5$.

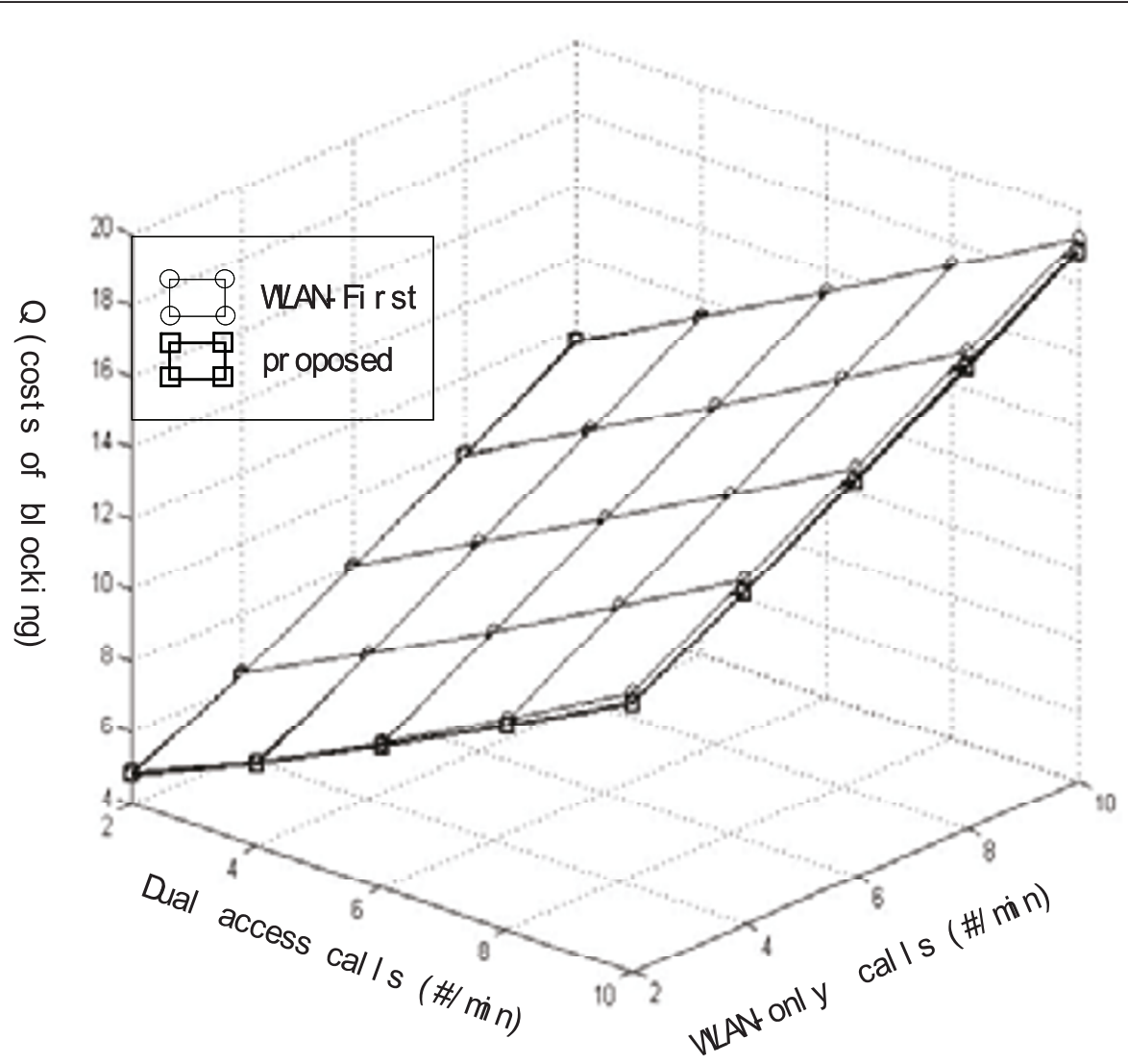

Figure 16 Results of guard band optimization with $\lambda_{c}^{d c a}=10, \lambda_{w c}^{c o a}=10, \lambda_{c}^{c o a}=10$ 
networks. The proposed scheme gives higher priority to the MSs with a single interface than MSs with dual interfaces by allocating a separate guard band for the MSs with a single interface. We analyze the performance of the proposed CAC scheme by means of Markov chains. Numerical results demonstrate that the proposed scheme achieves lower blocking probabilities than the WLAN-first scheme. Admission control for multi-service with heterogeneous MSs remains for further study.

\section{Acknowledgements}

This study was supported in part by the MKE, Korea, under the ITRC support program supervised by the NIPA (NIPA-2011-(C1090-1011-0004)), in part by the KCC, Korea, under the R\&D program supervised by the KCA (KCA-201110913-0500), and in part by the WCU Project (R33-2008-000-10044-0), and in part by the Basic Research Program (2009-0064397) through the NRF funded by the MEST, Korea.

\section{Competing interests}

The authors declare that they have no competing interests.

Received: 26 February 2011 Accepted: 6 September 2011

Published: 6 September 2011

\section{References}

1. E Gustafsson, A Jonsson, Always best connected. IEEE Wirel Commun. 10(2), 49-55 (2003)

2. V Varma, S Ramesh, K Wong, J Friedhoffer, Mobility management in integrated UMTS/WLAN networks, in Proc IEEE ICC, 1048-1053 (May2003)

3. M Buddhikot, G Chandranmenon, S Han, Y Lee, S Miller, L Salgarelli, Design and implementation of a WLAN/CDMA2000 internetworking architecture. IEEE Commun Mag. 41(11), 90-100 (2003). doi:10.1109/MCOM.2003.1244928

4. Chunanxioung Guo, Zhihua Guo, Qian Zhang, Wenwu Zhu, A seamless and proactive end-to-end mobility solution for roaming across heterogeneous wireless networks. IEEE J Sel Areas Commun. 22(5), 834-848 (2004). doi:10.1109/JSAC.2004.826921

5. Janise McNair, Fang Zhu, Vertical handoffs in fourth-generation multinetwork environments. IEEE Wirel Commun. 11(3), 8-15 (2004). doi:10.1109/MWC.2004.1308935

6. Thierry E Klein, Seung-Jae Han, Assignment strategies for mobile data users in hierarchical overlay networks: performance of optimal and adaptive strategies. IEEE J Sel Areas Commun. 22(5), 849-861 (2004). doi:10.1109/ JSAC.2004.826922

7. Wei Song, Hai Jiang, Weihua Zhuang, Performance analysis of the WLANfirst scheme in cellular/WLAN interworking. IEEE Trans Wirel Commun. 6(5), 1932-1952 (2007)

8. W Song, Y Cheng, W Zhuang, Improving voice and data services in cellular/ WLAN integrated networks by admission control. IEEE Trans Wirel Commun. 6(11), 4025-4037 (2007)

9. E Stevens-Navarro, A Hamed Mohesnian-Rad, WWS Wong, Connection admission control for multi-service integrated cellular/WLAN system. IEEE Trans Veh Technol. 57(6), 3789-3800 (2008)

10. LX Cai, X (Sherman) Shen, JW Mark, L Cai, Y Xiao, Voice capacity analysis of WLAN with unbalanced traffic. IEEE Trans Veh Technol. 55(3), 752-761 (2006). doi:10.1109/TVT.2006.874145

11. H Holma, A Toskala, WCDMA for UMTS, 3rd edn. (Wiley, 2004)

12. Y Fang, Modeling and performance analysis for wireless mobile networks: a new analytical approach. IEEE/ACM Trans Netw. 13(5), 989-1002 (2005)

13. C Blum, A Roli, Metaheuristics in combinatorial optimization: overview and conceptual comparison. ACM Comput Surv. 35(3), 268-308 (2003). doi:10.1145/937503.937505

\section{doi:10.1186/1687-1499-2011-91}

Cite this article as: Lim et al:: Call admission control with

heterogeneous mobile stations in cellular/WLAN interworking systems.

EURASIP Journal on Wireless Communications and Networking 2011 2011:91.

\section{Submit your manuscript to a SpringerOpen ${ }^{\mathcal{O}}$ journal and benefit from:}

- Convenient online submission

- Rigorous peer review

- Immediate publication on acceptance

- Open access: articles freely available online

- High visibility within the field

- Retaining the copyright to your article

Submit your next manuscript at $\gg$ springeropen.com 